\title{
FOURIER INVERSION OF INVARIANT INTEGRALS ON SEMISIMPLE REAL LIE GROUPS
}

\author{
BY
}

REBECCA A. HERB

\begin{abstract}
Let $G$ be a connected, semisimple real Lie group with finite center. Associated with every regular semisimple element $g$ of $G$ is a tempered invariant distribution $\Lambda_{g}$ given by an orbital integral. This paper gives an inductive formula for computing the Fourier transform of $\Lambda_{g}$ in terms of the space of tempered invariant eigendistributions of $G$.
\end{abstract}

1. Introduction. Let $G$ be a connected semisimple real Lie group with finite center. Let $g$ be the Lie algebra of $G, g_{C}$ its complexification. If $G_{C}$ is the simply connected complex analytic group corresponding to $g_{C}$, we assume that $G$ is the real analytic subgroup of $G_{\mathbf{C}}$ corresponding to $\mathrm{g}$.

Let $g$ be any regular semisimple element of $G$. Associated with $g$ is the tempered invariant distribution $\Lambda_{g}$ given by $f \mapsto \Lambda_{g}(f)=F_{f}^{H}(g), f \in C_{c}^{\infty}(G)$, where $H$ is the unique Cartan subgroup of $G$ containing $g$ and $F_{f}^{H}$ is the invariant integral of $f$ relative to $H$ defined by Harish-Chandra [1(a)].

The purpose of this paper is to give explicit formulas for the Fourier transform of $\Lambda_{g}$. That is, we determine a linear functional $\hat{\Lambda}_{g}$ such that $\Lambda_{g}(f)=\hat{\Lambda}_{g}(\hat{f}), f \in C_{c}^{\infty}(G)$. Here $\hat{f}$ is defined on the space of tempered invariant eigendistributions of $G$ which includes, for each conjugacy class [H] of Cartan subgroups of $G$, a series of tempered invariant eigendistributions of $G$ parameterized by the unitary character group $\hat{H}$ of $H$. These series include the characters of the discrete series representations of $G$ (if $G$ has a compact Cartan subgroup), the characters of the unitary principal series representations induced from each cuspidal parabolic subgroup of $G$, and certain "singular invariant eigendistributions" which can be interpreted as alternating sums of characters [3].

The Fourier inversion formula was computed in the case that $G$ has real rank one by P. Sally and G. Warner [6], and in the case that $G$ has real rank two by the author in her Ph. D. thesis [2], written under the supervision of G. Warner. In the real rank one case, Sally and Warner use the inversion formula to compute the Plancherel formula for G. Also, D. Ragozin and G. Warner have recently used the inversion formula for real rank one groups, together with the Selberg trace formula, to obtain information on multiplici-

Received by the editors October 5, 1977.

AMS (MOS) subject classifications (1970). Primary 22E30, 43A30. 
ties of irreducible representations of $G$ in $L^{2}(\Gamma \backslash G), \Gamma$ a co-compact discrete subgroup of $G$ [5].

The method of computing the Fourier transform for general groups is an extension of that used by Sally and Warner in the rank one case. However, there are two problems in computing Fourier inversion formulas for groups $G$ of real rank $n>1$ which do not occur when $n=1$, or when $n>1$ but $G$ has at most one conjugacy class of Cartan subgroups $[H]$ for each possible dimension of $H_{p}$, the vector part of $H$.

For $n=1$, a Fourier inversion formula can be obtained for any regular semisimple element of $G$. The same is true when $n=2$. However, there are cases when $n \geqslant 3$ (for example, $\operatorname{Sp}(3, \mathbf{R})$, the real symplectic group of rank three) in which certain integrals involved in computing the Fourier inversion formula for $F_{f}^{H}\left(h_{0}\right)$ diverge for certain regular elements $h_{0} \in H^{\prime}$. However, the Fourier inversion formula is still valid on a dense open set $H^{*}$ of $H$.

A second problem which occurs when $n \geqslant 2$ is that in the final formula for the Fourier inversion of $F_{f}^{H}\left(h_{0}\right)$, the coefficients of $\theta(f)$, for some tempered invariant eigendistributions $\theta$, are complicated expressions involving infinite sums which converge, but not absolutely, and have no obvious closed form. Thus they cannot be directly differentiated, term by term, to obtain a Plancherel formula for $G$. It is hoped, that in working with a specific group $G$ of rank $\geqslant 2$, the Fourier inversion formulas can be greatly simplified.

In $\S 2$, the series of tempered invariant eigendistributions associated with each Cartan subgroup of $G$ is described. In $\S 3$, Haar measures on $G$ and its subgroups are normalized, the invariant integral is defined, and formulas which will be needed in $\S 4$ are listed. For convenience, for many definitions and results we refer to [8]. In $\$ 4$ the main theorem of this paper, which can be used to obtain the complete inversion formula for $F_{f}^{H}\left(h_{0}\right), H$ any Cartan subgroup of $G, h_{0} \in H^{*}$, is proved. In $\S 5$, the proof of a technical lemma from $\S 2$ is given.

I am indebted to Paul Sally and Garth Warner for many helpful suggestions.

2. Tempered invariant eigendistributions on $G$. Let $G$ and $g$ be as in $\S 1$. Let $\mathfrak{g}=\mathfrak{l}+\mathfrak{p}$ be a Cartan decomposition of $\mathfrak{g}$ with Cartan involution $\theta$. Let $K$ be the maximal compact subgroup of $G$ corresponding to $f$. Let $H$ be a $\theta$-stable Cartan subgroup of $G$ with Lie algebra $\mathfrak{h}$. Then $\mathfrak{h}$ and $H$ have decompositions $\mathfrak{h}=\mathfrak{h}_{k}+\mathfrak{h}_{p}, \mathfrak{h}_{k}=\mathfrak{h} \cap \mathfrak{f}, \mathfrak{h}_{p}=\mathfrak{h} \cap \mathfrak{p}$, and $H_{K}=H_{K} H_{p}, H_{K}=H \cap K, H_{p}=$ $\exp \left(\mathfrak{h}_{p}\right)$.

Let $P \in \mathscr{P}\left(H_{p}\right)$, the set of parabolic subgroups of $G$ with split part $H_{p}$. Let $P=M H_{p} N$ be the Langlands decomposition of $P$. Let $L=M H_{p} . M$ and $L$ are reductive subgroups of $G$, but need not be connected or acceptable, and 
$H_{K}$ is a compact Cartan subgroup of $M$. (If $H=H_{K}$ is a compact Cartan subgroup of $G$, then $P=M=G$.)

To each unitary character of $H$ will be associated an invariant eigendistribution of $G$. These will include the characters of the principal series representations of $G$ induced from $P$, along with certain "singular characters".

Let $L_{H}=\left\{\lambda \in \sqrt{-1} \mathfrak{h}_{k}^{*} \mid \xi_{\lambda}(\exp H)=\exp (\lambda(H)), H \in \mathfrak{h}_{k}\right.$, extends to a well-defined character of $H_{K}^{0}$, the identity component of $H_{K}$ \}. $L_{H}$ is a lattice in $\sqrt{-1} \mathfrak{h}_{k}^{*}$. Corresponding to each $\lambda \in L_{H}$ there is an invariant eigendistribution $T(\lambda)$ defined on $M^{0}$, the identity component of $M$ [1(d), (e)]. If $\lambda \in L_{H}^{\prime}=\left\{\lambda \in L_{H} \mid\langle\lambda, \alpha\rangle \neq 0\right.$ for all $\left.\alpha \in \Phi\left(\mathfrak{m}_{\mathbf{c}}, \mathfrak{h}_{k} \mathbf{c}\right)\right\}$ where $\Phi\left(\mathfrak{m}_{\mathbf{c}}, \mathfrak{h}_{k} \dot{c}\right)$ denotes the set of roots of the complexified Lie algebra $\mathrm{m}_{\mathbf{c}}$ of $M$ with respect to $\mathfrak{h}_{k \mathrm{C}}$, then $T(\lambda)$ is, up to a sign, the character of a discrete series representation of $M^{0}$. If $\lambda \in L_{H}^{s}=L_{H} \backslash L_{H}^{\prime}, T(\lambda)$ is an alternating sum of characters which are "limits of discrete series" [3].

For any reductive group $G$ and Cartan subgroup $H$, define $W(G, H)=$ $N_{G}(H) / H$ where $N_{G}(H)$ is the normalizer of $H$ in $G$. For $G$ acceptable, define $\Delta_{H}^{G}$ as in $[8, \S 8.1]$. $M$ and $L$ need not be acceptable, but $M^{0}$ and $L^{0}$ are. If $J$ is a Cartan subgroup of $M$ or $L$ we will write $\Delta_{J}^{M}$ and $\Delta_{J}^{L}$ with the understanding that these are well-defined on $J \cap M^{0}$ and $J \cap L^{0}$ respectively.

Let $H^{\prime}=H \cap G^{\prime}$ where $G^{\prime}$ denotes the set of regular elements of $G$. Then the formulas for $T(\lambda)$ on $M^{0}$ are given as follows. (See [1(d)].)

$$
\begin{aligned}
& T(\lambda)\left(h_{k}\right)=\Delta_{H_{K}}^{M}\left(h_{k}\right)^{-1} \sum_{w \in W\left(M^{0}, H_{k}^{0}\right)} \operatorname{det} w \xi_{w \lambda}\left(h_{k}\right), \\
& h_{k} \in \dot{H_{k}^{\prime}} \cap M^{0} .
\end{aligned}
$$

Let $J=J_{K} J_{p}$ be a $\theta$-stable Cartan subgroup of $M^{0}$. Pick a connected component $J_{I}^{+}$of $J_{K}$. Let $k \in K \cap M^{0}$ satisfy $k J_{1}^{+} k^{-1} \subseteq H_{K}^{0}$. Let z denote the centralizer in $\mathrm{m}$ of ${J_{I}^{+}}^{+}, Z$ the connected subgroup of $M^{0}$ corresponding to z. Let $\mathfrak{i}^{\prime}(z)=\left\{H \in \mathfrak{i}_{p} \mid \alpha(H) \neq 0\right.$ for all $\left.\alpha \in \Phi\left(z_{c}, \dot{\mathrm{i}}_{c}\right)\right\}$. Let $\mathrm{i}_{p}^{+}$be a connected component of $\mathrm{i}^{\prime}(\mathrm{z}), J_{p}^{+}=\exp \left(\mathrm{i}_{p}^{+}\right)$. Let $y \in Z_{\mathrm{C}}$ satisfy $\operatorname{Ad}\left(y k^{-1}\right)\left(\mathfrak{h}_{k} \mathrm{C}\right)=$ $\dot{\mathrm{I}}_{\mathbf{C}}$. Let $j=j_{k} j_{p} \in J^{\prime}, j_{k} \in J_{I}^{+}, j_{p}=\exp \left(H_{p}\right) \in J_{p}^{+}$. Then

$$
\begin{aligned}
T(\lambda)\left(j_{k} j_{p}\right)= & \Delta_{J}^{M}\left(j_{k} j_{p}\right)^{-1} \sum_{t \in W\left({ }^{k} Z, H_{K}^{0}\right) \backslash W\left(M^{0}, H_{K}^{0}\right)} \operatorname{det} t \xi_{t \lambda}\left({ }^{k} j_{k}\right) \\
& \times \sum_{s \in W(Z, J)} \operatorname{det} s c_{\mathrm{z}}\left(s: t \lambda: \mathrm{i}_{p}^{+}\right) \exp \left(s^{y k^{-1}}(t \lambda)\left(H_{p}\right)\right) .
\end{aligned}
$$

The $c_{\mathrm{z}}\left(s: \lambda: \mathrm{i}_{p}^{+}\right)$are constants satisfying:

$$
c_{z}\left(s^{\prime} s: \lambda: s^{\prime} \mathrm{i}_{p}^{+}\right)=c_{z}\left(s: \lambda: \mathrm{i}_{p}^{+}\right), \quad s^{\prime} \in W(Z, J)
$$




$$
c_{\mathrm{z}}\left(s^{y k^{-1}} u: \lambda: \mathrm{i}_{p}^{+}\right)=c_{\mathrm{z}}\left(s: u \lambda: \mathrm{i}_{p}^{+}\right), \quad u \in W\left({ }^{k} Z, H_{K}^{0}\right) .
$$

Fix $J_{I}^{+}$and $\mathrm{i}_{p}^{+}$. Let $F_{\mathrm{z}}=\left\{{ }^{y k^{-1}} \lambda \mid \lambda \in \sqrt{-1} \mathrm{i}_{k}^{*}\right\}$ and $L_{\mathrm{z}}={ }^{y k^{-1}} L_{H}$. Set $F_{\mathrm{z}}^{\prime}=$ $\left\{\lambda \in F_{\mathrm{z}} \mid\langle\alpha, \lambda\rangle \neq 0\right.$ for all $\left.\alpha \in \Phi\left(\mathrm{z}_{\mathrm{c}}, \dot{\mathrm{\lambda}}_{\mathrm{C}}\right)\right\} . L_{\mathrm{z}}^{\prime}=L_{\mathrm{z}} \cap F_{\mathrm{z}}^{\prime}$ is called the set of regular elements of $L_{\mathrm{z}}, L_{\mathrm{z}}^{s}=L_{\mathrm{z}} \backslash L_{\mathrm{a}}^{\prime}$ the set of singular elements. For $\lambda \in L_{\mathrm{z}}$, we will write $c(s: \lambda)$ for $c_{z}\left(s:{ }^{k}{ }^{-} \lambda: \mathfrak{i}_{p}^{+}\right)$. Then for $\lambda \in L_{z}^{\prime}$, the constants $c(s: \lambda)$ are uniquely determined since the terms $\exp (s \lambda)$ are linearly independent on $J^{\prime}$ for $s \in W(Z, J)$. They depend only on the connected component $F^{+}$of $\lambda \in F_{z}^{\prime}$. Write $c(s: \lambda)=c\left(s: F^{+}\right)$when $\lambda \in F^{+}$.

If $\lambda \in L_{z}^{s}$, there exists some $s \neq 1$ in $W(Z, J)$ for which $s \lambda=\lambda$. Thus the constants $c(s: \lambda)$ are not uniquely determined. Let $F_{1}, \ldots, F_{k}$ be the connected components of $F_{z}^{\prime}$ with $\lambda \in \bar{F}_{i}, i=1, \ldots, k$. Then HarishChandra defines the constants $c(s: \lambda)$ by the average,

$$
c(s: \lambda)=\frac{1}{k} \sum_{i=1}^{k} c\left(s: F_{i}\right)
$$

[1(e)]. In order to prove convergence of certain integrals in $\$ 4$, it is necessary to make a different, but equivalent, definition for certain singular $\lambda$.

Let $\Phi^{+}=\left\{\alpha \in \Phi\left(z_{c}, \dot{i}_{c}\right) \mid \alpha(H)>0\right.$ for all $\left.H \in \mathrm{i}_{p}^{+}\right\}$. Let $\left\{\alpha_{1}, \ldots, \alpha_{l}\right\}$ be the set of simple roots for $\Phi^{+}$and $\left\{H_{1}, \ldots, H_{l}\right\}$ the dual basis of $\dot{\mathrm{x}}_{p}$ defined by $\alpha_{i}\left(H_{j}\right)=\delta_{i j}$. Then $\mathrm{i}_{p}^{+}=\left\{\sum_{i=1}^{l} r_{i} H_{i} \mid r_{i}>0, i=1, \ldots, l\right\}$. For $\lambda \in L_{z}, s \in$ $W(Z, J)$, write $s \lambda=\lambda_{I}(s \lambda)+\sum_{i=1}^{l} t_{i}(s \lambda) \alpha_{i}$ where $\lambda_{I}(s \lambda) \in \mathrm{i}_{C}^{*}$ assumes purely imaginary values on $\dot{\mathrm{j}}$, and $t_{i}(s \lambda)$ are real numbers, $1<i<l$. Then $c(s: \lambda)$ $=0$ if $\operatorname{Re}(s \lambda(H))>0$ for any $H \in \mathrm{i}_{p}^{+}[1(\mathrm{~d})]$, so $c(s: \lambda)=0$ if $t_{i}(s \lambda)>0$ for any $1 \leqslant i \leqslant l$.

LEMMA 2.5. Fix $\lambda_{0} \in L_{3}, s \in W(Z, J)$, and suppose $t_{i}(s \lambda)=0$ for some $1 \leqslant i \leqslant l$. Let $W^{\prime}=\left\{w \in W(Z, J) \mid w s \lambda_{0}=s \lambda_{0}\right\}$. Then

$$
\sum_{w \in W^{\prime}} \operatorname{det} w c\left(w s: \lambda_{0}\right)=0
$$

Since the proof of Lemma 2.5 is long and technical we defer it to $\$ 5$.

COROllary 2.6. Suppose $t_{i}(s \lambda)=0$ for any $1 \leqslant i \leqslant l$. Then if $\lambda$ is regular, $c(s: \lambda)=0$, and if $\lambda$ is singular, we may redefine $c(s: \lambda)=0$ without changing any of the properties of the constants $c(s: \lambda)$.

For $\lambda \in L_{H}^{\prime}$, let $\varepsilon(\lambda)=\operatorname{sign} \Pi_{\alpha \in \Phi^{+}\left(m_{c}, \mathfrak{h}_{k c}\right)}\langle\alpha, \lambda\rangle$. Let $q=\frac{1}{2} \operatorname{dim}(M / M \cap$ $K)$. Then for $\lambda \in L_{H}^{\prime},(-1)^{q} \varepsilon(\lambda) T(\lambda)$ is the character of a representation of the discrete series of $M^{0}$. For $\lambda \in L_{H}^{s}$, if $w \lambda=\lambda$ for $w \neq 1$ in $W\left(M^{0}, H_{K}^{0}\right)$, then $T(\lambda)=0$. Otherwise, $T(\lambda)$ is the alternating sum of characters which can be explicitly embedded in a certain reducible unitary principal series representation of $M^{0}$ [3]. 
Let $Z\left(\mathfrak{h}_{p}\right)=K \cap \exp \left(\sqrt{-1} \mathfrak{h}_{p}\right)$. This is a finite Abelian group generated by elements of order two, and $H_{K}=Z\left(\mathfrak{h}_{p}\right) H_{K}^{0}$. Define $M^{+}=Z\left(\mathfrak{h}_{p}\right) M^{0}$. Let $\rho\left(\mathfrak{m}, \mathfrak{h}_{k}\right)=\frac{1}{2} \sum \alpha, \alpha \in \Phi^{+}\left(\mathfrak{m}_{\mathrm{C}}, \mathfrak{h}_{k \mathfrak{K}}\right)$, and $\Gamma_{0}=Z\left(\mathfrak{h}_{p}\right) \cap H_{K}^{0}$. Let $\tilde{H}_{K}$ denote the set of all pairs $\lambda \in L_{H}$ and $\eta \in Z\left(\mathfrak{h}_{p}\right)^{\wedge}$ such that $\eta\left|\Gamma_{0}=\xi_{\lambda-\rho\left(m, b_{k}\right)}\right| \Gamma_{0}$. Then given $(\lambda, \eta) \in \tilde{H}_{K}$ there is an invariant eigendistribution $T(\lambda, \eta)$ on $M$ with support on $M^{+}$given by:

$$
\begin{aligned}
T(\lambda, \eta)(z m)=\eta(z) \sum_{\gamma \in M / M^{+}} T(\lambda)\left(\gamma m \gamma^{-1}\right), & \\
z & \in Z\left(\mathfrak{h}_{p}\right), \quad m \in M^{0} .
\end{aligned}
$$

(See [9].)

For any Cartan subgroup $J$ of $M$, representatives $\gamma$ for the cosets of $M / M^{+}$may be chosen so that $\gamma$ normalizes $J$ and centralizes $J_{p}$. In particular, for $h_{k} \in H_{K}^{\prime}, T(\lambda, \eta)$ has the simple formula

$$
\begin{aligned}
T(\lambda, \eta)\left(z h_{k}\right)=\eta(z) \Delta_{H_{K}}^{M}\left(h_{k}\right)^{-1} \sum_{w \in W\left(M, H_{K}\right)} \operatorname{det} w \xi_{w \lambda}\left(h_{k}\right), \\
z \in Z\left(\mathfrak{h}_{p}\right), h_{k} \in H_{K}^{0} .
\end{aligned}
$$

This formula is obtained from (2.7) using the fact that $W\left(M, H_{K}\right)$ is generated by $W\left(M^{0}, H_{K}^{0}\right)$ and conjugation by certain representatives of the cosets of $M / M^{+}$.

There is a one-to-one correspondence between pairs $(\lambda, \eta) \in \tilde{H}_{K}$ and elements $b^{*} \in \hat{H}_{K}$ given as follows. Elements of $\hat{H}_{K}$ are of the form $\chi \otimes \xi_{\lambda}$ where $\chi \in Z\left(\mathfrak{h}_{p}\right), \lambda \in L_{H}$, and $\chi\left|\Gamma_{0}=\xi_{\lambda}\right| \Gamma_{0}$. Let $\xi_{H}=\xi_{\rho(\mathfrak{g}, \mathfrak{h})}, \rho(\mathfrak{g}, \mathfrak{h})=\frac{1}{2} \Sigma \alpha$, $\alpha \in \Phi^{+}\left(g_{\mathbf{C}}, \mathfrak{h}_{\mathbf{C}}\right)$. If $\chi \otimes \xi_{\lambda} \in \hat{H}_{K}$, then $\left(\lambda, \xi_{H}^{-1} \otimes \chi\right) \in H_{K}$ as $\xi_{H}^{-1} \otimes \chi \mid \Gamma_{0}=$ $\xi_{\lambda-\rho(\mathfrak{g}, \mathrm{g})}\left|\Gamma_{0}=\xi_{\lambda-\rho\left(\mathrm{m}, \xi_{k}\right)}\right| \Gamma_{0}$. Conversely, if $(\lambda, \eta) \in \tilde{H}_{K}$, then $\chi=\eta \otimes \xi_{H} \in$ $Z\left(\mathfrak{h}_{p}\right)^{\wedge}$ and $\chi \otimes \xi_{\lambda} \in \hat{H}_{K}$. For $b^{*}=\chi \otimes \xi_{\lambda} \in \hat{H}_{K}$, we write $T\left(b^{*}\right)$ for $T\left(\lambda, \xi_{H}^{-1} \otimes \chi\right)$.

Each character of $H_{p}$ is of the form $h_{p}^{\vee(-1) r}=\exp \left(\sqrt{-1} \nu \log h_{p}\right), h_{p} \in$ $H_{p}, \nu \in \mathfrak{h}_{\mathfrak{R}}^{*}$, where log: $H_{p} \rightarrow \mathfrak{h}_{p}$ denotes the inverse of exp: $\mathfrak{h}_{p} \rightarrow H_{p}$. Given $(\lambda, \eta) \in H_{K}$ and $\nu \in \mathfrak{h}_{p}^{*}$, there is an invariant eigendistribution $\theta(H, \lambda, \eta, \nu)$ on $G$ with support on the closure of $\cup G^{J}, J$ a Cartan subgroup of $L$, where $G^{J}=\cup_{x \in G} x J^{\prime} x^{-1}$.

For any Cartan subgroup $J$ of $L$, let $J=J_{1}, J_{2}, \ldots, J_{k}$ be a complete set of representatives for distinct conjugacy classes of Cartan subgroups of $L$ for which $J_{i}$ is conjugate to $J$ in $G, i=1, \ldots, k$. (If $M$ is acceptable, $k$ is always one.) Let $x_{i} \in G$ satisfy $J_{i}=x_{i} J x_{i}^{-1}$ and for $j \in J$, write $j_{i}=x_{i} j x_{i}^{-1}$. Then for $j \in J^{\prime}$, 


$$
\begin{aligned}
\theta(H, \lambda, \eta, \nu)(j)= & \sum_{i=1}^{k}\left[W\left(L, J_{i}\right)\right]^{-1}\left|\Delta_{J_{i}}^{G}\left(j_{i}\right)\right|^{-1} \\
& \times \sum_{w \in W\left(G, J_{i}\right)}\left|\Delta_{J_{i}}^{L}\left(w j_{i}\right)\right| T(\lambda, \eta)\left(w j_{i} \mid M\right)\left(w j_{i} \mid H_{p}\right)^{\vee(-1) \nu},
\end{aligned}
$$

where for $j \in J_{i}=\left(J_{i} \cap M\right) H_{p}, j \mid M$ and $j \mid H_{p}$ denote the components of $j$ in $J_{i} \cap M$ and $H_{p}$ respectively (see [7]). Note that $\Delta_{J_{i}}^{L}$ is defined on $J_{i} \cap L^{0}$ only. However $\left|\Delta_{J_{i}}^{L}\right|$ makes sense on all of $J_{i}$. If $j \in J_{i}, j=z j^{0}, z \in Z\left(\mathfrak{h}_{p}\right), j^{0} \in J_{i} \cap$ $L^{0},\left|\Delta_{J_{i}}^{L}\left(z j^{0}\right)\right|$ is defined to be $\left|\Delta_{J_{i}}^{L}\left(j^{0}\right)\right|$. This is well defined as $\Delta_{J_{i}}^{L}\left(z j^{0}\right)=$ $\pm \Delta_{J_{i}}^{L}\left(j^{0}\right)$ for $z \in Z\left(\mathfrak{h}_{p}\right) \cap L^{0}$. For $b^{*}=\chi \otimes \xi_{\lambda} \in \hat{H}_{K}, \nu \in \mathfrak{h}_{p}^{*}$, write $\theta\left(H, b^{*}, \nu\right)$ for $\theta\left(H, \lambda, \xi_{H}^{-1} \otimes \chi, \nu\right)$.

When $\lambda \in L_{H}^{\prime},(-1)^{q} \varepsilon(\lambda) \theta(H, \lambda, \eta, \nu)$ is the character of a representation of the unitary principal series of $G$ induced from $P$. If $\lambda \in L_{H}^{s}, \theta(H, \lambda, \eta, \nu)$ is zero, or is an alternating sum of characters which can be embedded in a reducible unitary principal series representation associated to a different cuspidal parabolic subgroup [3].

3. The invariant integral. We must first normalize various invariant measures on $G$. For each Cartan subgroup $H$ of $G$, let $x \rightarrow \dot{x}$ denote the canonical projection of $G$ onto $G / H$. Normalize the $G$-invariant measure $d_{G / H}(\dot{x})$ on $G / H$ as in $[8, \S 8.1 .2]$. Let $H^{1}$ be a fundamental $\theta$-stable Cartan subgroup of $G$ with Cartan subalgebra $\mathfrak{h}^{1}$. Write $H^{1}=H_{K}^{1} H_{p}^{1}$. Normalize Haar measure on $H^{1}$ as follows. If $h=h_{k} h_{p} \in H^{1}, h_{k} \in H_{K}^{1}, h_{p} \in H_{p}^{1}$, let $d_{H^{\prime}}(h)=d_{H_{K}^{\prime}}\left(h_{k}\right) d_{H_{p}^{\prime}}\left(h_{p}\right), d_{H_{K}^{\prime}}$ the Haar measure on $H_{K}^{1}$ assigning it total mass one, $d_{H_{p}^{\prime}}$ the Haar measure on $H_{p}^{1}$ which is the transport via the exponential map of the canonical Haar measure on $\mathfrak{h}_{p}^{1}$ associated with the Euclidean structure derived from the Killing form on $G$. Then a Haar measure on $G$ is fixed by

$$
\int_{G} f(x) d_{G}(x)=\int_{G / H^{1}} \int_{H^{1}} f(x h) d_{H^{1}}(h) d_{G / H^{1}}(\dot{x})
$$

for all $f \in C_{c}(G)$.

A Haar measure on each $\theta$-stable Cartan subgroup $H$ of $G$ is then fixed by requiring that for all $f \in C_{c}(G)$,

$$
\int_{G} f(x) d_{G}(x)=\int_{G / H} \int_{H} f(x h) d_{H}(h) d_{G / H}(\dot{x}) .
$$

Write $H=H_{K} H_{p}$. Let $d_{H_{p}}$ be the Haar measure on $H_{p}$ which is the transport via the exponential map of the canonical Haar measure on its Lie algebra $\mathfrak{h}_{p}$. Normalize Haar measure on $H_{K}$ so that $d_{H}\left(h_{K} h_{p}\right)=d_{H_{K}}\left(h_{K}\right) d_{H_{p}}\left(h_{p}\right), h_{K} \in$ $H_{K}, h_{p} \in H_{p}$. Let $\operatorname{vol}\left(H_{K}\right)$ denote the total mass of $H_{K}$.

For any $\theta$-stable Cartan subgroup $H$ of $G, h=h_{K} h_{p} \in H^{\prime}, w \in W(G, H)$, 
define $\varepsilon_{R}^{H}(h), \varepsilon_{R}^{H}(w)$, and $\varepsilon_{W}^{H}\left(h_{k}\right)$ as in [8, §8.1.1]. For $f \in C_{c}^{\infty}(G), h \in H^{\prime}$, the invariant integral of $f$ relative to $H$ is defined by:

$$
F_{f}^{H}(h)=\varepsilon_{R}^{H}(h) \Delta_{H}^{G}(h) \int_{G / H} f\left(x h x^{-1}\right) d_{G / H}(\dot{x}) .
$$

Then $F_{f}^{H} \in L^{1}(H)$ and is $C^{\infty}$ on $H^{\prime}$. Further, there is a compact subset of $H$ off which $F_{f}^{H}$ vanishes. Let $G^{H}=\cup_{x \in G} x H^{\prime} x^{-1}$. Then from Weyl's formula it follows that

$$
\begin{aligned}
\int_{G^{H}} f(x) d_{G}(x)=[W(G, H)]^{-1} \int_{H} \varepsilon_{R}^{H}(h) \overline{\Delta_{H}^{G}(h)} F_{f}^{H}(h) d_{H}(h), & f \in C_{c}^{\infty}(G) .
\end{aligned}
$$

For $b^{*} \in \hat{H}_{K}, \nu \in \mathfrak{h}_{p}^{*}$, we define the Fourier transform of $F_{f}^{H}$ at $\left(b^{*}, \nu\right)$ by

$$
\hat{F}_{f}^{H}\left(b^{*}, \nu\right)=(2 \pi)^{-\operatorname{dim} \mathfrak{h}_{p} / 2} \int_{H_{K} \times H_{p}} F_{f}^{H}\left(h_{k} h_{p}\right) b^{*}\left(h_{k}\right) h_{p}^{\vee(-1) \nu} d_{H_{K}}\left(h_{k}\right) d_{H_{p}}\left(h_{p}\right) .
$$

Then since $F_{f}^{H} \in C^{\infty}\left(H^{\prime}\right)$, for $h=h_{k} h_{p} \in H^{\prime}$ we have

$$
F_{f}^{H}\left(h_{k} h_{p}\right)=(2 \pi)^{-\operatorname{dim} \mathfrak{h}_{p} / 2}\left(\operatorname{vol} H_{K}\right)^{-1} \sum_{b^{*} \in \hat{H}_{K}} \overline{b^{*}\left(h_{k}\right)} \int_{\mathfrak{h}_{p}^{*}} \hat{F}_{f}^{H}\left(b^{*}, \nu\right) h_{p}^{-v(-1) \nu} d \nu
$$

for a suitable normalization of Haar measure on $\mathfrak{h}_{p}^{*}$ and a suitable definition $\Sigma_{b^{*} \in \hat{H}_{K}}$ which will be made later.

We will need the following facts taken from [8, Chapter 8]. Let $w \in$ $W(G, H), h=h_{k} h_{p} \in H^{\prime}$. Define $M$ corresponding to $H$ as in $\$ 2$. Then:

$$
\begin{aligned}
\Delta_{H}^{G}(w h) & =\operatorname{det} w \Delta_{H}^{G}(h) ; \\
\varepsilon_{R}^{H}(w h) & =\varepsilon_{R}^{H}(w) \varepsilon_{w}^{H}\left(h_{k}\right) \varepsilon_{R}^{H}(h) ; \\
F_{f}^{H}(w h) & =\operatorname{det} w \varepsilon_{R}^{H}(w) \varepsilon_{w}^{H}\left(h_{k}\right) F_{f}^{H}(h) ; \\
\overline{\Delta_{H}^{G}(h)} & =(-1)^{r_{f}(H)} \Delta_{H}^{G}(h),
\end{aligned}
$$

where $r_{I}(H)$ is the number of elements of $\Phi^{+}\left(g_{C}, \mathfrak{h}_{C}\right)$ taking purely imaginary values on $\mathfrak{h}$.

Let $H_{I}^{+}$be a connected component of $H_{K}, z$ the centralizer in $g$ of $H_{I}^{+}$. Let $\Phi^{+}\left(\mathfrak{z}_{\mathbf{C}}, \mathfrak{h}_{\mathbf{C}}\right)=\Phi^{+}\left(\mathfrak{g}_{\mathbf{C}}, \mathfrak{h}_{\mathbf{C}}\right) \cap \Phi\left(\mathfrak{z}_{\mathbf{C}}, \mathfrak{h}_{\mathbf{C}}\right)$, and let $\mathfrak{h}_{p}^{+}=\left\{H \in \mathfrak{h}_{p} \mid \alpha(H)>0\right.$ for all $\left.\alpha \in \Phi^{+}\left(\mathfrak{z}_{\mathbf{C}}, \mathfrak{h}_{\mathbf{C}}\right)\right\}$. Then

$$
\varepsilon_{R}^{H}\left(h_{k} h_{p}\right)=1 \quad \text { for } h_{k} \in H_{I}^{+}, h_{p} \in \mathfrak{h}_{p}^{+} .
$$

Fix an ordering on the roots of $\Phi\left(g_{C}, \mathfrak{h}_{C}\right)$ so that for $\alpha \in \Phi\left(g_{C}, \mathfrak{h}_{C}\right)$ which does not take purely imaginary values on $\mathfrak{h}, \alpha$ is positive if and only if $\alpha^{\sigma}$ is positive. Hence $\alpha^{\sigma}(H)=\overline{\alpha(\sigma(H))}$ for all $H \in \mathfrak{h}_{\mathrm{C}}$, and $\sigma$ denotes the conju- 
gation of $g_{C}$ with respect to $\mathfrak{g}$. Let $\Phi^{+}\left(\mathfrak{m}_{\mathbf{C}}, \mathfrak{h}_{k \mathrm{C}}\right)=\Phi^{+}\left(\mathfrak{g}_{\mathbf{C}}, \mathfrak{h}_{\mathbf{C}}\right) \cap \Phi\left(\mathfrak{m}_{\mathbf{C}}, h_{k}\right)$. Then with respect to these orderings,

$$
\begin{array}{r}
\operatorname{sign}\left\{\frac{\Delta_{H}^{G}\left(\gamma h_{k} h_{p}\right)}{\Delta_{H_{K}}^{M}\left(h_{k}\right)}\right\}=\varepsilon_{R}^{H}\left(\gamma h_{k} h_{p}\right) \xi_{H}(\gamma) \\
\qquad \text { for } \gamma \in Z\left(\mathfrak{h}_{p}\right), h_{k} \in\left(H_{K}^{0}\right)^{\prime}, h_{p} \in H_{p} .
\end{array}
$$

Here $\xi_{H}=\xi_{\rho(\mathfrak{g}, \mathfrak{h})}$ as in \$2. Further, if $J$ is any Cartan subgroup of $L$, $J_{M}=J \cap M$, then for $\gamma \in Z\left(\mathfrak{h}_{p}\right), j_{M} \in J^{\prime} \cap M^{0}, h_{p} \in H_{p}$,

$$
\operatorname{sign}\left\{\frac{\Delta_{J}^{G}\left(\gamma j_{M} h_{p}\right)}{\Delta_{J_{M}}^{M}\left(j_{M}\right)}\right\}=\varepsilon_{R}^{J}\left(\gamma j_{M} h_{p}\right) \varepsilon_{R}^{J_{M}}\left(j_{M}\right) \xi_{J}(\gamma)
$$

Here $\xi_{J}=\xi_{\rho(g, i)}$ and $\varepsilon_{R}^{J_{M}}$ is defined with respect to $M$ and $J_{M}$. Again, $\Phi^{+}\left(g_{C}, \dot{\mathrm{l}}_{C}\right)$ is chosen so that for $\alpha \in \Phi\left(g_{C}, \dot{\mathrm{l}}_{C}\right), \alpha$ not taking purely imaginary values on $i, \alpha$ is positive if and only if $\alpha^{\sigma}$ is positive. $\Phi^{+}\left(m_{C}, \dot{i}_{M_{C}}\right)=$ $\Phi^{+}\left(\mathrm{g}_{\mathbf{C}}, \mathrm{i}_{\mathbf{C}}\right) \cap \Phi\left(\mathrm{m}_{\mathbf{C}}, \mathrm{i}_{M_{C}}\right)$.

4. The Fourier inversion formula. Fix a $\boldsymbol{\theta}$-stable Cartan subgroup $\boldsymbol{H}$ of $\boldsymbol{G}$. Let $M$ be as in $\S 2$. Let $\operatorname{Car}(G, H)$ be a complete set of $\theta$-stable representatives for the $G$-conjugacy classes of Cartan subgroups of $L=M H_{p}$. Let $\operatorname{Car}^{\prime}(G, H)=\operatorname{Car}(G, H) \backslash\{H\}$. Let $\xi_{H}$ and $r_{I}(H)$ be as defined in (3.10) and (3.8) respectively. Set $d\left(H_{p}\right)=\operatorname{dim} \mathfrak{h}_{p}$.

LemMa 4.1. Let $f \in C_{c}^{\infty}(G), b^{*}=\chi \otimes \xi_{\lambda} \in \hat{H}_{K}, \nu \in \mathfrak{h}_{p}^{*}$. Then $\hat{F}_{f}^{H}\left(b^{*}, \nu\right)$

$$
\begin{aligned}
=\frac{(-1)^{r_{I}(H)}}{(2 \pi)^{d\left(H_{p}\right) / 2}\{\Theta}\left(H, \lambda, \chi \otimes \xi_{H}^{-1}, \nu\right)(f) \\
\\
\left.\quad-\sum_{J \in \operatorname{Car}^{\prime}(G, H)} \int_{G^{J}} \Theta\left(H, \lambda, \chi \otimes \xi_{H}^{-1}, \nu\right)(x) f(x) d_{G}(x)\right\} .
\end{aligned}
$$

Proof. Since $\theta=\theta\left(H, \lambda, \chi \otimes \xi_{H}^{-1}, \nu\right)$ has support on $\cup_{J \in \operatorname{Car}(G, H)} G^{J}$,

$$
\theta(f)=\int_{G} \theta(x) f(x) d_{G}(x)=\sum_{J \in \operatorname{Car}(G, H)} \int_{G^{J}} \theta(x) f(x) d_{G}(x)
$$

Using (3.2), (3.8), (2.9), (3.10), (2.8), and (3.3) respectively, we have 


$$
\begin{aligned}
\int_{G^{H}} \theta(x) f(x) d_{G}(x)= & {[W(G, H)]^{-1} \int_{H} \varepsilon_{R}^{H}(h) \overline{\Delta_{H}^{G}(h)} F_{f}^{H}(h) \theta(h) d_{H}(h) } \\
= & (-1)^{r_{f}(H)}\left[W\left(M, H_{K}\right)\right]^{-1}[W(G, H)]^{-1} \sum_{w \in W(G, H)} \\
& \times \int_{H_{K} \times H_{p}} \varepsilon_{R}^{H}\left(h_{k} h_{p}\right) \Delta_{H}^{G}\left(h_{k} h_{p}\right) F_{f}^{H}\left(h_{k} h_{p}\right) \frac{\left|\Delta_{H_{K}}^{M}\left(h_{k}\right)\right|}{\left|\Delta_{H}^{G}\left(h_{k} h_{p}\right)\right|} \\
& \times T\left(\lambda, \chi \otimes \xi_{H}^{-1}\right)\left(w h_{k}\right)\left(w h_{p}\right)^{V(-1) \nu} d_{H_{K}}\left(h_{k}\right) d_{H_{p}}\left(h_{p}\right) \\
= & (-1)^{r_{f}(H)}\left[W\left(M, H_{K}\right)\right]^{-1} \sum_{\gamma \in Z^{\prime}} \int_{H_{K}^{0} \times H_{p}} F_{f}^{H}\left(\gamma h_{k} h_{p}\right) \\
& \times\left(\sum_{w \in W\left(M, H_{K}\right)} \operatorname{det} w \xi_{\lambda}\left(w h_{k}\right)\right) \chi(\gamma) h_{p}^{\vee(-1) v} d_{H_{k}}\left(h_{k}\right) d_{H_{p}}\left(h_{p}\right) \\
= & (-1)^{r_{f}(H)}(2 \pi)^{d\left(H_{p}\right) / 2} \hat{F}_{f}^{H}\left(b^{*}, \nu\right),
\end{aligned}
$$

where $Z^{\prime}=Z\left(\mathfrak{h}_{p}\right) / Z\left(\mathfrak{h}_{p}\right) \cap H_{K}^{0}$. Q.E.D.

In order to consider the Fourier series of $F_{f}^{H}$, we must make precise the type of convergence used relative to $\hat{H}_{K}$. Let $\left\{\beta_{1}, \ldots, \beta_{r}\right\}$ be the set of simple roots of $\Phi^{+}\left(\mathfrak{m}_{\mathbf{c}}, \mathfrak{h}_{k \mathcal{C}}\right)$. Let $\left\{\Lambda_{1}, \ldots, \Lambda_{r}\right\}$ be the basis of $\sqrt{-1} \mathfrak{h}_{k}^{*}$, satisfying $2\left\langle\Lambda_{i}, \beta_{j}\right\rangle /\left\langle\beta_{j}, \beta_{j}\right\rangle=\delta_{i j}, 1<i, j<r$. Then $L_{H}=\left\{\sum_{i=1}^{r} m_{i} \Lambda_{i} \mid m_{i} \in\right.$ $Z$ \}. For any positive integer $M$, define $L_{H}^{M}=\left\{\sum_{i=1}^{r} m_{i} \Lambda_{i} \mid-M<m_{i}<M\right.$, $1<i<r\}$. Given $\chi \in Z\left(\mathfrak{h}_{p}\right)^{\wedge}$, let $L_{\chi}=\left\{\lambda \in L_{H}\left|\xi_{\lambda}\right|_{\Gamma_{0}}=\left.\chi\right|_{\Gamma_{0}}\right\}, \Gamma_{0}=Z\left(\mathfrak{h}_{p}\right) \cap$ $H_{K}^{0}$. Let $L_{\chi}^{M}=L_{\chi} \cap L_{H}^{M}$. Then summability relative to $H_{K}$ is defined by

$$
\sum_{b^{*} \in \hat{H}_{K}}=\lim _{M \rightarrow \infty} \sum_{x \in Z\left(\mathfrak{h}_{p}\right)^{-}} \sum_{\lambda \in L_{\chi}^{M}} .
$$

LEMMA 4.2. Let $h_{0}=h_{k} h_{p} \in H^{\prime}$. Then

$$
\begin{aligned}
F_{f}^{H}\left(h_{0}\right)= & \frac{(-1)^{r_{l}(H)}}{\operatorname{vol} H_{K}(2 \pi)^{d\left(H_{p}\right)}} \sum_{b^{*} \in \hat{H}_{K}} \overline{b^{*}\left(h_{k}\right)} \\
& \times \int_{\mathfrak{h}_{p}^{*}} h_{p}^{-V(-1) \nu} \theta\left(H, b^{*}, \nu\right)(f) d \nu+I_{f}^{H}\left(h_{0}\right),
\end{aligned}
$$

where

$$
\begin{aligned}
& I_{f}^{H}\left(h_{0}\right)=\frac{(-1)^{r_{f}(H)+1}}{\operatorname{vol} H_{K}(2 \pi)^{d\left(H_{p}\right)}} \sum_{b^{*} \in \hat{H}_{K}} \overline{b^{*}\left(h_{k}\right)} \int_{\mathfrak{h}_{p}^{*}} h_{p}^{-V(-1) \nu} \\
& \quad \times\left\{\sum_{J \in \operatorname{Car}^{\prime}(G, H)} \int_{G^{J}} \theta\left(H, b^{*}, \nu\right)(x) f(x) d_{G}(x)\right\} d \nu .
\end{aligned}
$$


Proof. Use (3.4) and Lemma 4.1. Since

$$
\sum_{b^{*} \in \hat{H}_{K}} \overline{b^{*}\left(h_{k}\right)} \int_{\mathfrak{h}_{p}^{*}} h^{-v(-1) \nu} \theta\left(H, b^{*}, \nu\right)(f) d \nu
$$

converges absolutely the result is clear [1(d)], [9]. Q.E.D.

The remainder of $\S 4$ is devoted to an analysis of $I_{f}^{H}\left(h_{0}\right)$. The main result of this paper is that there is a dense open set $H^{*} \subseteq H^{\prime}$ such that if $h_{0} \in H^{*}$, then for $J=J_{K} J_{p} \in \operatorname{Car}^{\prime}(L, H)$ (see Lemma 4.3),

$$
\sum_{b^{*} \in \hat{H}_{K}} \overline{b^{*}\left(h_{k}\right)} \int_{\mathfrak{h}_{p}^{*}} h_{p}^{-\vee(-1) \nu} \int_{G^{J}} \theta\left(H, b^{*}, \nu\right)(x) f(x) d_{G}(x) d \nu
$$

can be expressed as a finite number of terms of the form

$$
\sum_{b^{*} \in \hat{J}_{K}} \overline{b^{*}\left(j_{k}\right)} \int_{\mathrm{i}_{p}^{*}} g\left(j_{p}, \mu\right) \hat{F}_{f}^{J}\left(b^{*}, \mu\right) d \mu
$$

where $j_{0}=j_{k} j_{p}$ depends on $h_{0} \in H^{*}$ and $g\left(j_{p}, \mu\right)$ is a continuous function of $j_{p}$ and $\mu$.

Let $Q=M_{1} J_{p} N_{1} \in \mathcal{P}\left(J_{p}\right)$. If $J$ is a Cartan subgroup of $G$ with $\operatorname{dim} J_{p}$ maximal, then $Q$ is a minimal parabolic subgroup of $G, M_{1}$ is compact, and $J_{K}$ is, up to conjugacy, its only Cartan subgroup. Thus

$$
\hat{F}_{f}^{J}\left(b^{*}, \mu\right)=\frac{(-1)^{r_{t}(J)}}{(2 \pi)^{d\left(J_{p}\right) / 2}} \theta\left(J, b^{*}, \mu\right)(f)
$$

is, up to scalar multiple, the character of a unitary principal series representation of $G$ induced from the minimal parabolic subgroup $Q$.

Otherwise, if $\operatorname{dim} J_{p}$ is not maximal, let $\operatorname{Car}(G, J)$ be a complete set of $\theta$-stable representatives for $G$-conjugacy classes of Cartan subgroups of $L_{1}=$ $M_{1} J_{p}, \operatorname{Car}^{\prime}(G, J)=\operatorname{Car}(G, J) \backslash\{J\}$. In this case, by applying Lemma (4.1) to $J$,

$$
\begin{aligned}
\hat{F}_{f}^{J}\left(b^{*}, \mu\right)=\frac{(-1)^{r_{l}(J)}}{(2 \pi)^{d\left(J_{p}\right) / 2}}\left\{\theta\left(J, b^{*}, \mu\right)(f)\right. & \\
& \left.-\sum_{A \in \operatorname{Car}^{\prime}(G, J)} \int_{G^{A}} \theta\left(J, b^{*}, \mu\right)(x) f(x) d_{G}(x)\right\},
\end{aligned}
$$

and so, as in Lemma 4.2,

$$
\begin{aligned}
\sum_{b^{*} \in \hat{J}_{K}} \overline{b^{*}\left(j_{k}\right)} \int_{\mathrm{i}_{p}^{*}} g\left(j_{p}, \mu\right) \hat{F}_{f}^{J}\left(b^{*}, \mu\right) d \mu \\
=\frac{(-1)^{r_{f}(J)}}{(2 \pi)^{d\left(J_{p}\right) / 2}} \sum_{b^{*} \in \hat{J}_{K}} \overline{b^{*}\left(j_{k}\right)} \int_{\mathrm{i}_{p}^{*}} g\left(j_{p}, \mu\right) \theta\left(J, b^{*}, \mu\right)(f) d \mu+I_{f}^{J}\left(j_{0}\right),
\end{aligned}
$$


where

$$
\begin{aligned}
I_{f}^{J}\left(j_{0}\right)=\frac{(-1)^{r_{t}(J)+1}}{(2 \pi)^{d\left(J_{p}\right) / 2}} \sum_{b^{*} \in \hat{J}_{K}} \overline{b^{*}\left(j_{k}\right)} \\
\quad \times \int_{\mathrm{i}_{p}^{*}} g\left(j_{p}, \mu\right)\left\{\sum_{A \in \operatorname{Car}^{\prime}(G, J)} \int_{G^{A}} \theta\left(J, b^{*}, \mu\right)(x) f(x) d_{G}(x)\right\} d \mu .
\end{aligned}
$$

Clearly each of the terms $I_{f}^{J}\left(j_{0}\right)$ can be evaluated in the same manner as $I_{f}^{H}\left(h_{0}\right)$. Further, $I_{f}^{H}\left(h_{0}\right)$ involved terms corresponding to Cartan subgroups with $\operatorname{dim} J_{p}>\operatorname{dim} H_{p}$. Each $I_{f}^{J}\left(j_{0}\right)$ involves only terms corresponding to Cartan subgroups $A \in \operatorname{Car}^{\prime}(G, J)$ for which $\operatorname{dim} A_{p}>\operatorname{dim} J_{p}$. Thus the process will terminate in a finite number of steps.

Fix a Cartan subgroup $J$ of $L$. Write $J=J_{M} H_{p} . J_{M}=J \cap M$ is a Cartan subgroup of $M$ with Cartan decomposition $J_{M}=J_{K} J_{M, p}$. Write $A=J_{M, p}$. Define $d_{A}$ on $A$ to be the transport via the exponential map of the canonical Haar measure on $\mathfrak{a}$, the Lie algebra of $A$. Then since $\mathfrak{i}_{p}=\mathfrak{h}_{p}+\mathfrak{a}$ is an orthogonal direct sum, $d_{J_{p}}\left(h_{1} h_{2}\right)=d_{A}\left(h_{1}\right) d_{H_{p}}\left(h_{2}\right), h_{1} \in A, h_{p} \in H_{p}$.

Let $\Gamma_{1}$ be a set of representatives in $Z\left(\mathfrak{h}_{p}\right)$ for $Z\left(\mathfrak{h}_{p}\right) / Z\left(\mathfrak{h}_{p}\right) \cap J_{K}^{0}$. Let $\Gamma_{2}$ be a set of representatives in $Z(\mathfrak{a})$ for $Z(\mathfrak{a}) / Z(\mathfrak{a}) \cap Z\left(\mathfrak{h}_{p}\right) J_{K}^{0}$. Let $\Gamma_{3}$ be a set of representatives for $M / M^{+}$which normalize $J_{M}$ and centralize $J_{p}$.

Note that $Z\left(\mathfrak{h}_{p}\right)$ and $Z(\mathfrak{a})$ are subgroups of the abelian group $Z\left(\dot{\mathfrak{d}}_{p}\right)$ and so elements of $\Gamma_{1}, \Gamma_{2}$ and $\Gamma_{3}$ commute. Normalize Haar measure on $J_{K}^{0}$ so that for $f \in C\left(J_{K} \cap M^{+}\right)$,

$$
\int_{J_{K} \cap M^{+}} f\left(j_{k}\right) d_{J_{K}}\left(j_{k}\right)=\sum_{\gamma_{1} \in \Gamma_{1}} \sum_{\gamma_{2} \in \Gamma_{2}} \int_{J_{K}^{0}} f\left(\gamma_{1} \gamma_{2} j_{k}\right) d_{J_{K}^{0}}\left(j_{k}\right) .
$$

We will simplify notation by always writing $j=\gamma_{1} \gamma_{2} j_{k} h_{1} h_{2}$ for $\gamma_{1} \in \Gamma_{1}$, $\gamma_{2} \in \Gamma_{2}, j_{k} \in J_{K}^{0}, h_{1} \in A$ and $h_{2} \in H_{p}$, and abbreviating $d_{J_{K}^{0}}\left(j_{k}\right), d_{A}\left(h_{1}\right)$, and $d_{H_{p}}\left(h_{2}\right)$ by $d j_{k}, d h_{1}$, and $d h_{2}$ respectively.

LEMMA 4.3. Let $\operatorname{Car}(L, H)$ be a set of representatives for the $L$-conjugacy classes of Cartan subgroups of $L, \operatorname{Car}^{\prime}(L, H)=\operatorname{Car}(L, H) \backslash\{H\}$. Then

$$
\begin{aligned}
I_{f}^{H}\left(h_{0}\right)= & \frac{(-1)^{r_{I}(H)+1}\left[\Gamma_{3}\right]}{\operatorname{vol} H_{K}(2 \pi)^{d\left(H_{p}\right)}} \sum_{b^{*} \in \hat{H}_{K}} \overline{b^{*}\left(h_{k}\right)} \int_{\mathfrak{h}_{p}^{*}} h_{p}^{-V(-1) \nu} \\
& \times\left\{\sum_{J \in \operatorname{Car}^{\prime}(L, H)} \frac{(-1)^{r_{t}(J)}}{\left[W\left(M, J_{M}\right)\right]} \sum_{\gamma_{1} \in \Gamma_{1}} \sum_{\gamma_{2} \in \Gamma_{2}} I_{f}^{H}\left(J, b^{*}, \nu, \gamma_{1}, \gamma_{2}\right)\right\} d \nu
\end{aligned}
$$


where

$$
\begin{aligned}
I_{f}^{H}\left(J, b^{*}, \nu, \gamma_{1}, \gamma_{2}\right)=\int_{J_{K}^{0} \times A \times H_{p}} \varepsilon_{R}^{J_{M}} & \left(\gamma_{2} j_{k} h_{1}\right) F_{f}^{J}\left(\gamma_{1} \gamma_{2} j_{k} h_{1} h_{2}\right) \Delta_{J_{M}}^{M}\left(\gamma_{2} j_{k} h_{1}\right) \\
& \times \chi\left(\gamma_{1}\right) T(\lambda)\left(\gamma_{2} j_{k} h_{1}\right) h_{2}^{V(-1) \nu} d j_{k} d h_{1} d h_{2} .
\end{aligned}
$$

Proof. Using (3.2), (2.9), (3.5), and (3.11),

$$
\begin{aligned}
& \sum_{J \in \operatorname{Car}^{\prime}(G, H)} \int_{G^{J}} \theta\left(H, b^{*}, v\right)(x) f(x) d_{G}(x) \\
& =\sum_{J \in \operatorname{Car}^{\prime}(G, H)} \frac{(-1)^{r_{t}(J)}}{[W(G, J)]} \int_{J} \varepsilon_{R}^{J}(j) \Delta_{J}^{G}(j) F_{f}^{J}(j) \theta\left(H, b^{*}, \nu\right)(j) d J(j) \\
& =\sum_{J \in \operatorname{Car}^{\prime}(L, H)} \frac{(-1)^{r,(J)}}{[W(G, J)]} \sum_{w \in W(G, J)} \int_{J} \varepsilon_{R}^{J}(j) \frac{\Delta_{J}^{G}(j)}{\left|\Delta_{J}^{G}(j)\right|} \\
& \times F_{f}^{J}(j) \frac{\left|\Delta_{J}^{L}(w j)\right|}{[W(L, J)]} T\left(b^{*}\right)(w j \mid M)\left(w j \mid H_{p}\right)^{\vee(-1) v} d j \\
& =\sum_{J \in \operatorname{Car}^{\prime}(L, H)} \frac{(-1)^{r_{t}(J)}}{\left[W\left(M, J_{M}\right)\right]} \sum_{\gamma_{1} \in \Gamma_{1}} \sum_{\gamma_{2} \in \Gamma_{2}} \\
& \times \int_{J_{K}^{0} \times A \times H_{p}} \varepsilon_{R}^{J_{M}}\left(\gamma_{2} j_{k} h_{1}\right) F_{f}^{J}\left(\gamma_{1} \gamma_{2} j_{k} h_{1} h_{2}\right) \Delta_{J_{M}}^{M}\left(\gamma_{2} j_{k} h_{1}\right) \\
& \times \chi\left(\gamma_{1}\right) h_{2}^{\gamma^{(-1)} \nu} \sum_{\gamma_{3} \in \Gamma_{3}} T(\lambda)\left(\gamma_{3} \gamma_{2} j_{k} h_{1} \gamma_{3}^{-1}\right) d j_{k} d h_{1} d h_{2} \\
& =\sum_{J \in \operatorname{Car}^{\prime}(L, H)} \frac{(-1)^{r_{1}(J)}\left[\Gamma_{3}\right]}{\left[W\left(M, J_{M}\right)\right]} \sum_{\gamma_{1} \in \Gamma_{1}} \sum_{\gamma_{2} \in \Gamma_{2}} I_{f}^{H}\left(J, b^{*}, \nu, \gamma_{1}, \gamma_{2}\right) \text {. }
\end{aligned}
$$

To expand the sum over $\operatorname{Car}^{\prime}(G, H)$ to a sum over $\operatorname{Car}^{\prime}(L, H)$ we use (2.9) and the fact that for $J_{i}=x_{i} J x_{i}^{-1}, x_{i} \in G, j_{i}=x_{i} j x_{i}^{-1}$ for $j \in J, \varepsilon_{R}^{J_{i}}\left(j_{i}\right)=$ $\varepsilon_{R}^{J}(j), \Delta_{J_{i}}^{G}\left(j_{i}\right)=\Delta_{J}^{G}(j)$, and $F_{f}^{J_{i}}\left(j_{i}\right)=F_{f}^{J}(j)$. Q.E.D.

Fix $J \in \operatorname{Car}^{\prime}(L, H), b^{*}=\xi_{\lambda} \otimes \chi \in \hat{H}_{K}, \nu \in \mathfrak{h}_{p}^{*}, \gamma_{1} \in \Gamma_{1}$, and $\gamma_{2} \in \Gamma_{2}$. Write $I\left(\gamma_{1}, \gamma_{2}\right)$ for $I_{f}^{H}\left(J, b^{*}, \nu, \gamma_{1}, \gamma_{2}\right)$. Define $z, Z, k$, and $y$ as in $\S 2$ with respect to $J_{I}^{+}=\gamma_{2} J_{K}^{0}$. Use $a^{*}$ to denote the component of $h_{1}$ in $\dot{i}_{M}^{\prime}(\mathrm{z})$. Let $\mathfrak{a}^{+}$ 
denote the positive chamber of $\mathfrak{i}_{M}^{\prime}(\mathrm{z})$ with respect to $\Phi^{+}\left(z_{C}, \dot{\mathrm{I}}_{M C}\right)=$ $\Phi^{+}\left(\mathrm{g}_{C}, \dot{\mathrm{j}}_{\mathrm{C}}\right) \cap \Phi\left(\mathrm{z}_{\mathrm{C}}, \dot{\mathrm{i}}_{M C}\right)$. Write $A^{+}=\exp \left(\mathrm{a}^{+}\right)$. Let $W_{M}=W\left(M^{0}, H_{K}^{0}\right), W_{Z}$ $=W\left({ }^{-1} Z, H_{K}^{0}\right)$. Then using (2.2), (3.5), (3.6), (3.7) (2.3) and (3.9),

$$
\begin{aligned}
& I\left(\gamma_{1}, \gamma_{2}\right)=\int_{J_{K}^{0} \times A \times H_{p}} \varepsilon_{R}^{J_{M}}\left(\gamma_{2} j_{k} h_{1}\right) F_{f}^{J}\left(\gamma_{1} \gamma_{2} j_{k} h_{1} h_{2}\right) \chi\left(\gamma_{1}\right) h_{2}^{\gamma^{(-1) \nu}} \\
& \times \sum_{t \in W_{Z} \backslash W_{M}} \operatorname{det} t \xi_{k^{-1}(t \lambda)}\left(\gamma_{2} j_{k}\right) \sum_{s \in W\left(Z, J_{M}\right)} \operatorname{det} s c_{\mathrm{z}}\left(s: t \lambda: a^{*}\right) \\
& \times \exp \left(s^{y k^{-1}}(t \lambda)\left(\log h_{1}\right)\right) d j_{k} d h_{1} d h_{2} \\
& =\left[W\left(Z, J_{M}\right)\right] \sum_{t \in W_{Z} \backslash W_{M}} \operatorname{det} t \int_{J_{K}^{0} \times A \times H_{p}} \varepsilon_{R}^{J_{M}}\left(\gamma_{2} j_{k} h_{1}\right) F_{f}^{J}(j) \\
& \times \chi\left(\gamma_{1}\right) h_{2}^{\gamma(-1) v} \xi_{k^{-1}(t \lambda)}\left(\gamma_{2} j_{k}\right) \\
& \times c_{\mathrm{z}}\left(I: t \lambda: a^{*}\right) \exp \left(s^{y k^{-1}}(t \lambda)\left(\log h_{1}\right)\right) d j_{k} d h_{1} d h_{2} \\
& =\left[W\left(Z, J_{M}\right)\right] \sum_{t \in W_{Z} \backslash W_{M}} \operatorname{det} t \sum_{s \in W\left(Z, J_{M}\right)} \operatorname{det} s \\
& \times \int_{J_{K}^{0} \times A^{+} \times H_{p}} F_{f}^{J}\left(\gamma_{1} \gamma_{2} j_{k} h_{1} h_{2}\right) \chi\left(\gamma_{1}\right) h_{2}^{\gamma(-1) v} \xi_{k^{-1}(t \lambda)}\left(\gamma_{2} j_{k}\right) \\
& \times c_{\mathrm{z}}\left(s: t \lambda: \mathfrak{a}^{+}\right) \exp \left(s^{y k^{-1}}(t \lambda)\left(\log h_{1}\right)\right) d j_{k} d h_{1} d h_{2} \text {. }
\end{aligned}
$$

By definition,

$$
{ }^{y k^{-1}} W_{Z}={ }^{y k^{-1}} W\left({ }^{k^{-1}} Z, H_{K}^{0}\right) \subseteq W\left(z_{c},{ }^{y k^{-1}} \mathfrak{h}_{k \mathrm{c}}\right)=W\left(Z, J_{M}\right)
$$

Thus using (2.4) we can write

$$
\begin{aligned}
I\left(\gamma_{1}, \gamma_{2}\right)=\sum_{t \in W_{M}} \operatorname{det} t \int_{J_{K}^{0} \times A^{+}} \times H_{p} F_{f}^{J}\left(\gamma_{1} \gamma_{2} j_{k} h_{1} h_{2}\right) h_{2}^{\gamma(-1) v} \chi\left(\gamma_{1}\right) \\
\quad \times \xi_{k^{-1}(t \lambda)}\left(\gamma_{2} j_{k}\right) s\left(\gamma_{2}: t \lambda: h_{1}\right) d j_{k} d h_{1} d h_{2}
\end{aligned}
$$

where

$$
\begin{aligned}
s\left(\gamma_{2}: \lambda: h_{1}\right)=[ & \left.W\left(Z, J_{M}\right)\right] \sum_{s \in W\left(Z, J_{M}\right) /{ }^{y k^{-1}} W_{Z}} \operatorname{det} s c_{\mathrm{z}}\left(s: \lambda: \mathrm{a}^{+}\right) \\
& \times \exp \left(s^{y k^{-1}} \lambda\left(\log h_{1}\right)\right) .
\end{aligned}
$$

Fix $M>0 . L_{\chi}$ is $W\left(M^{0}, H_{K}^{0}\right)$-stable. If $t \in W\left(M^{0}, H_{K}^{0}\right)$, write $t \cdot L_{\chi}^{M}=$ $\left\{t \lambda \mid \lambda \in L_{x}^{M}\right\}$. Then 


$$
\begin{aligned}
& \sum_{\chi \in Z\left(\mathfrak{h}_{p}\right)^{-}} \sum_{\lambda \in L_{\chi}^{M}} \overline{\left(\chi \otimes \xi_{\lambda}\right)\left(h_{k}\right)} \int_{\mathfrak{h}_{p}^{*}} h_{p}^{-\vee(-1) \nu} \\
& \times \int_{G^{J}} \theta\left(H, \lambda, \chi \otimes \xi_{H}^{-1}, \nu\right)(x) f(x) d_{G}(x) d \nu \\
& =\frac{(-1)^{r_{t}(J)}\left[M / M^{+}\right]}{\left[W\left(M, J_{M}\right)\right]} \sum_{t \in W\left(M^{0}, H_{K}^{0}\right)} \operatorname{det} t \\
& \times \sum_{\gamma_{2} \in \Gamma_{2}} \sum_{\gamma_{1} \in \Gamma_{1}} \sum_{\chi \in Z\left(\mathfrak{h}_{p}\right)^{)}} \sum_{\lambda \in t^{-1} L_{\chi}^{M}} \overline{\left(\chi \otimes \xi_{\lambda}\right)\left(t^{-1} h_{k}\right)} \\
& \times \int_{\mathfrak{h}_{p}^{*}} h_{p}^{-\vee(-1) \nu} \int_{J_{K}^{0} \times A^{+} \times H_{p}} F_{f}^{J}\left(\gamma_{1} \gamma_{2} j_{k} h_{1} h_{2}\right) h_{2}^{(-1) \nu} \\
& \times \chi\left(\gamma_{1}\right) \xi_{k^{-1}(t \lambda)}\left(\gamma_{2} j_{k}\right) s\left(\gamma_{2}: \lambda: h_{1}\right) d j_{k} d h_{1} d h_{2}
\end{aligned}
$$

Fix $\gamma_{2} \in \Gamma_{2}$ and use the notation of (2.2) for $J_{I}^{+}=\gamma_{2} J_{K}^{0}$. Then $k^{-1} H_{K}^{0}=J_{K}^{0} \exp \left(y^{-1} \sqrt{-1}\right.$ a). Write $k^{-1} H_{k}^{0}=T, J_{K}^{0}=T_{1}$ and $\exp \left(\left(^{y^{-1}} \sqrt{-1} a\right)=T_{2}\right.$. Let $L^{*}=\left\{\left.\lambda \in{ }^{k^{-1}} L_{H}\right|^{y} \lambda \mid \mathfrak{a}=0\right.$ and $\left.\left.\xi_{k_{\lambda}}\right|_{\Gamma_{0}}=1\right\}$. Let $L_{p}=\left\{\sum_{i=1}^{l} n_{i} \alpha_{i} \mid n_{i} \in Z, 1 \leqslant i \leqslant l\right\}$ where $\left\{\alpha_{1}, \ldots, \alpha_{l}\right\}$ is a set of simple roots for $\Phi\left(z_{c}, \dot{\mathrm{I}}_{M C}\right)$. Let $L_{0}=L^{*} \oplus^{y^{-1}} L_{p} . L^{*}$ and $y^{-1} L_{p}$ parameterize the character groups of $T_{1} / T_{1} \cap \Gamma_{0} T_{2}$ and $T_{2} / T_{2} \cap C(Z)$ respectively, where $C(Z)$ denotes the center of $Z .{ }^{k^{-1}} L / L_{0}$ has finite order. Pick representatives $\tau_{1}, \ldots, \tau_{m} \in k^{-1} L$ of the cosets ${ }^{k^{-1}} L / L_{0}$. Then every element $\lambda_{0}$ of $L_{H}$ can be written uniquely as $\lambda_{0}={ }^{k}\left(\tau^{*}+{ }^{y^{-1}} \lambda+\tau_{i}\right), \tau^{*} \in L^{*}, \lambda \in L_{p}, 1<i<m$, and the consistency condition $\left.\xi_{\lambda_{0}}\right|_{\Gamma_{0}}=\left.\chi\right|_{\Gamma_{0}}$ is equivalent to $\xi_{k_{i}}\left|\Gamma_{0}=\chi\right|_{\Gamma_{0}}$. Let $Z\left(\mathfrak{h}_{p}\right)_{i}^{\hat{i}}=\left\{x \in Z\left(\mathfrak{h}_{p}\right)^{\wedge}|\chi|_{\Gamma_{0}}=\xi_{k_{k_{i}}} \Gamma_{\Gamma_{0}}\right\}$.

Write $h_{k}=\gamma_{0} h_{k}^{0}, \quad \gamma_{0} \in Z\left(\mathfrak{h}_{p}\right), h_{k}^{0} \in H_{K}^{0}$. For $t \in W\left(M^{0}, H_{K}^{0}\right)$, write $k^{-1}\left(t^{-1} h_{k}^{g}\right)=j_{1}(t) j_{2}(t)$, where $j_{1}(t) \in T_{1}, j_{2}(t)=\exp \left(y^{-1}\left(-\sqrt{-1} J_{2}(t)\right)\right) \in$ $T_{2}, J_{2}(t) \in a$. Using the above decompositions of $\lambda_{0}$ and $t^{-1} h_{k}$, we have:

$$
\begin{aligned}
\overline{\chi \otimes \xi_{\lambda_{0}}\left(t^{-1} h_{k}\right)} & =\chi\left(\gamma_{0}\right){\overline{\xi_{r^{*}+\tau_{i}}\left(j_{1}(t)\right) e}}^{V(-1)\left(\lambda+{ }^{\tau_{i}}\right)\left(J_{2}(t)\right)} \\
\xi_{k^{-1} \lambda_{0}}\left(\gamma_{2} j_{k}\right) & =\xi_{\gamma_{\tau_{i}}}\left(\gamma_{2}\right) \xi_{r^{*}+\tau_{i}}\left(j_{k}\right) \\
s\left(\gamma_{2}: \lambda_{0}: h_{1}\right) & =s\left(\gamma_{2}:{ }^{k y^{-1}} \lambda+{ }^{k} \tau_{i}: h_{1}\right)
\end{aligned}
$$

Thus for fixed $t \in W\left(M^{0}, H_{K}^{0}\right), \gamma_{2} \in \Gamma_{2}$, with the understanding that all sums are restricted to $t^{-1} L_{H}^{M}$, 


$$
\begin{aligned}
& \sum_{\chi \in Z\left(\mathfrak{h}_{p}\right)^{\circ}} \sum_{\lambda_{0} \in t^{-1} L_{\chi}^{M}} \overline{\left(\chi \otimes \xi_{\lambda_{0}}\right)\left(t^{-1} h_{k}\right)} \int_{\mathfrak{h}_{p}^{*}} h_{p}^{-V(-1) \nu} \\
& \times \sum_{\gamma_{1} \in \Gamma_{1}} \int_{J_{K}^{0} \times A^{+} \times H_{p}} F_{f}^{J}\left(\gamma_{1} \gamma_{2} h_{1} h_{2}\right) h_{2}^{\gamma(-1) v} \chi\left(\gamma_{1}\right) \\
& \times \xi_{k^{-1}(\lambda)}\left(\gamma_{2} j_{k}\right) s\left(\gamma_{2}: \lambda: h_{1}\right) d j_{k} d h_{1} d h_{2} \\
& =\sum_{i=1}^{m} \xi_{\gamma_{i}}\left(\gamma_{2}\right) \int_{\mathfrak{h}_{p}^{*}} h_{p}^{-V(-1) \nu} \int_{A^{+}} \sum_{\lambda \in L_{p}} e^{v(-1)\left(\lambda+{ }^{\gamma} \tau_{i}\right)\left(J_{2}(t)\right)} s\left(\gamma_{2}:{ }^{k y^{-1}} \lambda+{ }^{k} \tau_{i}: h_{1}\right) \\
& \times \int_{H_{p}} h_{2}^{\gamma(-1) \nu} \sum_{\chi \in Z\left(b_{p}\right) \hat{i}} \chi\left(\gamma_{0}\right) \sum_{\tau^{*} \in L^{*}} \overline{\xi_{r^{*}+\tau_{i}}\left(j_{1}(t)\right)} \sum_{\gamma_{1} \in \Gamma_{1}} \chi\left(\gamma_{1}\right) \\
& \times \int_{J_{K}^{0}} \xi_{r^{*}+\tau_{i}}\left(j_{k}\right) F_{f}^{J}\left(\gamma_{1} \gamma_{2} j_{k} h_{1} h_{2}\right) d j_{k} d h_{1} d h_{2} d \nu .
\end{aligned}
$$

LeMma 4.10. Fix $i=1, \ldots, m$. Then, if the sum is taken over all of $L^{*}$,

$$
\begin{gathered}
\sum_{x \in Z\left(\mathfrak{b}_{p}\right)_{i}} \chi\left(\gamma_{0}\right) \sum_{\tau^{*} \in L^{*}} \overline{\xi_{\tau^{*}+\tau_{i}}\left(j_{1}(t)\right)} \sum_{\gamma_{1} \in \Gamma_{1}} \chi\left(\gamma_{1}\right) \int_{J_{K}^{\sigma_{*}^{*}+\tau_{i}}}\left(j_{k}\right) F_{f}^{J}\left(\gamma_{1} \gamma_{2} j_{k} h_{1} h_{2}\right) d j_{k} \\
=\frac{\left(\operatorname{vol} J_{K}^{0}\right)\left[\Gamma_{1}\right]}{\left[\Gamma_{4}\right]} \sum_{\gamma_{4} \in \Gamma_{4}} \xi_{r_{i}}\left(\gamma_{4}\right) F_{f}^{J}\left(\gamma_{2} \gamma_{4} \gamma_{0} j_{1}(t) h_{1} h_{2}\right),
\end{gathered}
$$

where $\Gamma_{4}=Z\left(\mathfrak{h}_{p}\right) J_{K}^{0} \cap \Gamma_{0} Z(\mathfrak{a})$. The sum over $L^{*}$ converges uniformly for $h_{1} h_{2} \in A H_{p}$ and uniformly for compacta of $j_{1}(t) \in\left(J_{K}^{0}\right)^{\prime}$, but not absolutely.

Proof. Fix $h_{1} h_{2} \in A H_{p}$, and $\chi_{i} \in Z\left(\mathfrak{h}_{p}\right)_{i}$. For $j_{k} \in Z\left(\mathfrak{h}_{p}\right) J_{K}^{0}$, define

$$
g\left(j_{k}\right)=\sum_{\gamma_{4} \in \Gamma_{4}}\left(\chi_{i} \otimes \xi_{r_{i}}\right)\left(\gamma j_{k}\right) F_{f}^{J}\left(\gamma_{2} \gamma j_{k} h_{1} h_{2}\right) \text {. }
$$

Then $g$ is well defined on $Z\left(\mathfrak{h}_{p}\right) J_{K}^{0} / \Gamma_{4}$, and by elementary Fourier analysis,

$$
\begin{aligned}
& \sum_{x \in Z\left(\mathfrak{h}_{p}\right)_{i}} \chi\left(\gamma_{0}\right) \sum_{\tau^{*} \in L^{*}} \overline{\xi_{r^{*}+\tau_{i}}\left(j_{1}(t)\right)} \sum_{\gamma_{1} \in \Gamma_{1}} \chi\left(\gamma_{1}\right) \int_{J_{k}^{0}} \xi_{\tau^{*}+\tau_{i}}\left(j_{k}\right) F_{f}^{J}\left(\gamma_{1} \gamma_{2} j_{k} h_{1} h_{2}\right) d j_{k} \\
& =\frac{1}{\left[\Gamma_{4}\right]} \overline{\left(\chi_{i} \otimes \xi_{r_{i}}\right)\left(\gamma_{0} j_{1}(t)\right)} \sum_{x \in\left(z\left(\mathfrak{(}_{p}\right) / \Gamma_{0}\right)^{-}} x\left(\gamma_{0}\right) \\
& \times \sum_{\tau^{*} \in L^{*}} \overline{\xi_{r^{*}}\left(j_{1}(t)\right)} \int_{Z\left(\mathfrak{h}_{p}\right) J_{K}^{0} / \Gamma_{4}}\left(\chi \otimes \xi_{r^{*}}\right)\left(j_{k}\right) g\left(j_{k}\right) d \overline{j_{k}} \\
& =\frac{1}{\left[\Gamma_{4}\right]} \overline{\left(x_{i} \otimes \xi_{r_{i}}\right)\left(\gamma_{0} j_{1}(t)\right)} \sum_{b^{*} \in\left(z\left(\mathfrak{h}_{p}\right) J_{K}^{0} / \Gamma_{4}\right)^{*}} \overline{b^{*}\left(\gamma_{0} j_{1}(t)\right)}
\end{aligned}
$$




$$
\begin{aligned}
& \times \int_{Z\left(\mathfrak{h}_{p}\right) J_{K}^{0} / \Gamma_{4}} b^{*}\left(j_{k}\right) g\left(j_{k}\right) d \overline{j_{k}} \\
= & \frac{\left[\Gamma_{1}\right]\left(\operatorname{vol} J_{K}^{0}\right)}{\left[\Gamma_{4}\right]} \overline{\chi_{i} \otimes \xi_{\tau_{i}}\left(\gamma_{0} j_{1}(t)\right)} g\left(\gamma_{0} j_{1}(t)\right) \\
= & \frac{\left[\Gamma_{1}\right]\left(\operatorname{vol} J_{K}^{0}\right)}{\left[\Gamma_{4}\right]} \sum_{\gamma_{4} \in \Gamma_{4}} \xi_{\tau_{i}}\left(\gamma_{4}\right) F_{f}^{J}\left(\gamma_{2} \gamma_{1} \gamma_{0} j_{1}(t) h_{1} h_{2}\right) .
\end{aligned}
$$

Here $d \overline{j_{k}}$ on $Z\left(\mathfrak{h}_{p}\right) J_{K}^{0} / \Gamma_{4}$ is defined by

$$
\begin{aligned}
\sum_{\gamma_{1} \in \Gamma_{1}} \int_{J_{K}^{0}} f\left(\gamma_{1} j_{k}\right) d j_{k}=\frac{1}{\left[\Gamma_{4}\right]} \sum_{\gamma_{4} \in \Gamma_{4}} \int_{Z\left(\mathfrak{h}_{p}\right) J_{K}^{0} / \Gamma_{4}} f\left(\gamma_{4} j_{k}\right) d j_{k} & \text { for } f \in C\left(Z\left(\mathfrak{h}_{p}\right) J_{K}^{0}\right) \text {. Q.E.D. }
\end{aligned}
$$

LEMMA 4.11. There is a dense open set $T_{2}^{*}$ of $T_{2}$ such that

$$
\left|\sum_{\substack{\lambda \in L_{p} \ni \\ y^{-1} \lambda+\tau_{i} \in k^{-1}\left(t^{-1} \cdot L_{H}^{M}\right)}} e^{V(-1)\left(\lambda+y_{i}\right)\left(J_{2}(t)\right)} s\left(\gamma_{2}: k y^{-1} \lambda+{ }^{k} \tau_{i}: h_{1}\right)\right|
$$

is bounded independent of $M$, uniformly for $h_{1} \in A^{+}$, and for $j_{2}(t)={ }^{y}\left(\exp -\sqrt{-1} J_{2}(t)\right)$ in compact subsets of $T_{2}^{*}$.

Proof. $L_{p} \subseteq \mathrm{i}_{M}^{*}=F_{2}$. Let $F_{2}^{\prime}=\left\{\lambda \in F_{2} \mid\langle\alpha, \lambda\rangle \neq 0, \alpha \in \Phi\left(z_{c}, \dot{\mathrm{i}}_{M c}\right)\right\}$. Let $C_{1}, \ldots, C_{k}$ be the chambers of $F_{2}^{\prime}$ for which $C_{j} \subseteq F_{-}=\left\{\lambda \in F_{2} \mid \lambda(H)<0\right.$ for all $\left.H \in \overline{\mathfrak{a}}^{+}, H \neq 0\right\}, j=1, \ldots, k$. These are the chambers of $F_{2}^{\prime}$ on which $c_{z}\left(s: k y^{-1}\left(s^{-1} \lambda\right): \mathfrak{a}^{+}\right)=c\left(s: s^{-1} C_{j}\right)$ is constant, $\lambda \in C_{j}$, and possibly nonzero. In addition, we need to consider a finite number of "singular chambers" of the form $C_{\lambda_{0}}=\left\{\lambda \in F_{2} \mid \lambda \in \bar{C}^{+}\right.$if and only if $\lambda_{0} \in \bar{C}^{+}$for every chamber $C^{+}$of $\left.F_{2}^{\prime}\right\}$, for fixed singular $\lambda_{0} \in L_{p}$. Let $C_{k+1}, \ldots, C_{r}$ be the distinct "singular chambers" for which $C_{j} \subseteq F_{-}, j=k+1, \ldots, r$. Then $c_{f}\left(s: k y^{-1}\left(s^{-1} \lambda\right): a^{+}\right)=c\left(s: s^{-1} C_{j}\right)$ is constant for $\lambda \in C_{j}, k+1<j<r$. Further, $c_{z}\left(s:{ }^{k y^{-1}}\left(s^{-1} \lambda\right): a^{+}\right)=0$ if $\lambda \notin \cup_{j=1}^{r} C_{j}$. Then, all sums being restricted to ${ }^{y^{-1}} \lambda+\tau_{i} \in{ }^{k}\left(t^{-1} \cdot L_{H}^{M}\right)$,

$$
\begin{aligned}
& \sum_{\lambda \in L_{p}} e^{V(-1)\left(\lambda+{ }^{y} \tau_{i}\right)\left(J_{2}(t)\right)} \sum_{s \in W\left(Z, J_{M}\right) / y k^{-1} W_{Z}} \operatorname{det} s c_{z}\left(s:{ }^{k y-1} \lambda+{ }^{k} \tau_{i}: a^{+}\right) \\
& \times \exp \left(s\left(\lambda+{ }^{y} \tau_{i}\right)\left(\log h_{1}\right)\right) \\
& =\sum_{s \in W\left(Z, J_{M}\right) / y k^{-1} W_{Z}} \operatorname{det} s \sum_{i=1}^{r} c\left(s: s^{-1} C_{j}\right) \sum_{\substack{\lambda \in L_{p} \ni \\
\lambda+\gamma_{\tau_{i}} \in C_{j}}} e^{\left(\lambda+\gamma_{\tau_{i}}\right)\left(V(-1) s^{-1} J_{2}(t)+\log h_{1}\right)} .
\end{aligned}
$$


Fix $s \in W\left(Z, J_{M}\right)$ and $1 \leqslant j \leqslant r$. Write $\log h_{1}+\sqrt{-1} s^{-1} J_{2}(t)=H$. Then

$$
\sum_{\substack{\lambda \in L_{p} \ni \\ \lambda+\gamma_{\tau_{i}} \in C_{j}}} e^{\left(\lambda+\gamma_{\tau_{i}}\right)(H)}=e^{\nu_{\tau_{i}}(H)} \sum_{\substack{\lambda \in L_{p} \ni \\ \lambda+\nu \tau_{i} \in C_{j}}} e^{\lambda(H)}
$$

is just a geometric sum over a lattice in a convex subset of $F_{2}$, and its absolute value can be bounded by a finite number of terms (depending on $C_{j}$, but not on $M$ ) of the form

$$
\frac{2\left|e^{\lambda_{0}(H)}\right|}{\left|1-e^{\lambda_{1}(H)}\right| \cdots\left|1-e^{\lambda_{3}(H)}\right|}
$$

where $\lambda_{0} \in C_{j} \cap F_{-}$and where $\lambda_{i}, 1 \leqslant i \leqslant s \leqslant l$, are integral linear combinations of $\alpha_{1}, \ldots, \alpha_{l}$. Let $\Lambda\left(J, \gamma_{2}, t\right)$ denote the finite set of $\lambda_{i}$ which arise in the denominators obtained from the geometric sums over $C_{j}, 1<j<$ $r$. Define $T_{2}^{*}\left(J, \gamma_{2}, t\right)=\left\{e^{-\vee(-1)^{y} H} \in T_{2} \mid H \in a, e^{\vee(-1) \lambda\left(s^{-1} H\right)} \neq 1, s \in\right.$ $\left.W\left(Z, J_{M}\right), \lambda \in \Lambda\left(J, \gamma_{2}, t\right)\right\}$. Then if $\left|1-\cos \lambda\left(s^{-1} J_{2}(t)\right)\right|>\varepsilon$ for all $s \in$ $W\left(Z, J_{M}\right), \lambda \in \Lambda\left(J, \gamma_{2}, t\right)$, we have

$$
\frac{2\left|e^{\lambda_{0}(H)}\right|}{\left|1-e^{\lambda_{1}(H)}\right| \cdots\left|1-e^{\lambda_{s}(H)}\right|} \leqslant \frac{2}{\varepsilon^{s}}
$$

since $\operatorname{Re}\left(\lambda_{0}(H)\right)=\lambda_{0}\left(\log h_{1}\right)<0, \lambda_{0} \in F_{-}, h_{1} \in A^{+}$. Q.E.D.

Let $H^{*}=\left\{h \in H^{\prime} \mid\right.$ for every $J \in \operatorname{Car}^{\prime}(L, H), t \in W\left(M^{0}, H_{K}^{0}\right), \gamma_{2} \in \Gamma_{2}$, in the decomposition ${ }^{k^{-1}}\left(t^{-1} h_{k}^{9}\right)=j_{1}(t) j_{2}(t), j_{1}(t) \in T_{1}, j_{2}(t) \in T_{2}$ used in (4.9), we have $\left.j_{2}(t) \in T_{2}^{*}\left(J, \gamma_{2}, t\right)\right\}$.

LEMMA 4.12.

$$
\begin{aligned}
I_{f}^{H}\left(h_{0}\right)= & \frac{(-1)^{r_{I}(H)+1}\left[M / M^{+}\right]}{\operatorname{vol} H_{K}(2 \pi)^{d\left(H_{p}\right)}} \sum_{J \in \operatorname{Car}^{\prime}(L, H)} \frac{(-1)^{r_{t}(J)}}{\left[W\left(M, J_{M}\right)\right]} \\
& \times \sum_{t \in W\left(M^{0}, H_{K}^{0}\right)} \operatorname{det} t \sum_{\gamma_{2} \in Z(\mathrm{a}) \Gamma_{0}} I_{f}^{H}\left(J, t, \gamma_{2}, h_{0}\right)
\end{aligned}
$$

where

$$
\begin{aligned}
& I_{f}^{H}\left(J, t, \gamma_{2}, h_{0}\right) \\
& =\int_{\mathfrak{h}_{p}^{*}} h_{p}^{-V(-1) \nu} \int_{A^{+}} \sum_{i=1}^{m} \xi_{\gamma_{i}}\left(\gamma_{2}\right) \sum_{\lambda \in L_{p}} e^{v(-1)\left(\lambda+\gamma_{\tau_{i}}\right)\left(J_{2}(t)\right)} s\left(\gamma_{2}: k y^{-1} \lambda+{ }^{k} \tau_{i}: h_{1}\right) \\
& \quad \times \int_{H_{p}} h_{2}^{\gamma(-1) \nu} \frac{\left(\operatorname{vol} J_{K}^{0}\right)\left[\Gamma_{1}\right]}{\left[\Gamma_{4}\right]} F_{f}^{J}\left(\gamma_{2} \gamma_{0} j_{1}(t) h_{1} h_{2}\right) d h_{2} d h_{1} d \nu .
\end{aligned}
$$


Proof. By Lemmas 4.10 and 4.11, the sums over $L^{*}$ and $L_{p}$ are bounded independent of $M$, uniformly in $\nu, h_{1}$, and $h_{2}$. Thus the $\lim _{M \rightarrow \infty}$ can be taken inside of each of the integrals. The sums over $\Gamma_{2}=Z(\mathfrak{a}) / Z(\mathfrak{a}) \cap Z\left(\mathfrak{h}_{p}\right) J_{K}^{0}$ and $\Gamma_{4}=\Gamma_{0} Z(\mathfrak{a}) \cap Z\left(\mathfrak{h}_{p}\right) J_{K}^{0}=\Gamma_{0}\left(Z(\mathfrak{a}) \cap Z\left(\mathfrak{h}_{p}\right) J_{K}^{0}\right)$ have been combined to yield a sum over $Z(a) \Gamma_{0}$. Q.E.D.

Using (3.3), (3.4), we have that for $h_{1} \in A, j_{k} \in J_{K}$,

$$
\begin{aligned}
\int_{H_{p}} h_{2}^{\gamma(-1) \nu} F_{f}^{J}\left(j_{k} h_{1} h_{2}\right) d h_{2} & \\
& =(2 \pi) \frac{\left(d\left(H_{p}\right)-d(A)\right) / 2}{\operatorname{vol} J_{K}} \sum_{b^{*} \in \hat{J}_{K}} \overline{b^{*}\left(j_{k}\right)} \int_{\mathfrak{a}^{*}} h_{1}^{-v(-1) \mu} \hat{F}_{f}^{J}\left(b^{*}, \mu \otimes \nu\right) d \mu
\end{aligned}
$$

where the sum over $b^{*} \in \hat{J}_{K}$ converges uniformly on compacta of $J_{K}^{\prime}$, but not absolutely, and the integral over $a^{*}$ converges absolutely. The integral

$$
\begin{aligned}
I_{J}^{H}\left(\gamma_{2}: J_{2}(t), \mu\right)= & \int_{A^{+}} \sum_{i=1}^{m} \xi_{\gamma_{i}}\left(\gamma_{2}\right) \sum_{\lambda \in L_{p}} e^{V(-1)\left(\lambda+\gamma_{\tau_{i}}\right)\left(J_{2}(t)\right)} \\
& \times s\left(\gamma_{2}: k y^{-1} \lambda+{ }^{k} \tau_{i}: h_{1}\right) h_{1}^{-V(-1) \mu} d h_{1}
\end{aligned}
$$

converges absolutely also, since the absolute value of the integrand is bounded by a finite number of terms of the form $c e^{\lambda_{0}\left(\log h_{1}\right)}, c$ a constant (varying uniformly with $e^{-V(-1)^{y} J_{2}(t)}$ in compacta of $T_{2}^{*}$ ) and $\lambda_{0} \in F_{-}$. (Here

$$
\int_{A^{+}} e^{\lambda_{0}\left(\log h_{1}\right)} d h_{1}=c_{A} \int_{0}^{\infty} \cdots \int_{0}^{\infty} e^{-n_{1} r_{1}-\cdots-n_{l} r_{l}} d r_{1} \cdots d r_{l}
$$

where $n_{i}>0$ for $1 \leqslant i \leqslant l, c_{A}$ a constant relating $d h_{1}$ to $d r_{1} \cdots d r_{l}$.) Thus we may use (4.13) and then exchange the order of integration to obtain:

$$
\begin{aligned}
I_{f}^{H}\left(J, t, \gamma_{2}, h_{0}\right)= & (2 \pi) \frac{\left(d\left(H_{p}\right)-d(A)\right) / 2}{\left[Z(\mathfrak{a}) \cdot \Gamma_{0}\right]\left[Z(\mathfrak{a}) \cap Z\left(\mathfrak{h}_{p}\right)\right]} \\
& \times \int_{\mathfrak{h}_{p}^{*}} h_{p}^{-v(-1) \nu} \int_{\mathfrak{a}^{*}} I_{J}^{H}\left(\gamma_{2}: J_{2}(t), \mu\right) \\
& \times \sum_{b^{*} \in \hat{J}_{K}} \overline{b^{*}\left(\gamma_{0} \gamma_{2} j_{1}(t)\right)} \hat{F}_{f}^{J}\left(b^{*}, \mu \otimes \nu\right) d \mu d \nu
\end{aligned}
$$


THeOREM 4.15. Let $h_{0}=h_{k} h_{p} \in H^{*}$. Then

$$
\begin{aligned}
F_{f}^{H}\left(h_{0}\right)= & \frac{(-1)^{r_{1}(H)}}{\operatorname{vol} H_{K}(2 \pi)^{\operatorname{dim}\left(h_{p}\right)}} \sum_{b^{*} \in \hat{H}_{K}} \overline{b^{*}\left(h_{k}\right)} \int_{\mathfrak{h}_{p}^{*}} h_{p}^{-v(-1) v} \theta\left(H, b^{*}, \nu\right)(f) d \nu \\
& +\frac{(-1)^{r_{t}(H)+1}\left[M / M^{+}\right]}{\operatorname{vol} H_{K}} \\
& \times \sum_{J \in \operatorname{Car}^{\prime}(L, H)} \frac{1}{(2 \pi)^{\operatorname{dim} i_{p}}\left[W\left(M, J_{M}\right)\right]\left[Z(a) \cdot \Gamma_{0}\right]\left[Z(a) \cap Z\left(\mathfrak{h}_{p}\right)\right]} \\
& \times \sum_{t \in W\left(M^{0}, H_{K}^{0}\right)} \operatorname{det} t \sum_{\gamma_{2} \in Z(a) \Gamma_{0}} \\
& \times\left[\sum_{b^{*} \in \hat{J}_{K}} \frac{b^{*}\left(\gamma_{0} \gamma_{2} j_{1}(t)\right)}{\int_{\mathfrak{h}_{p}^{*}}} h_{p}^{-V(-1) \nu} \int_{\mathfrak{a}^{*}} I_{J}^{H}\left(\gamma_{2}: J_{2}(t): \mu\right)\right.
\end{aligned}
$$$$
\left.\times \theta\left(J, b^{*}, \mu \otimes \nu\right)(f) d \mu d \nu+I_{J}^{H}\left(f, h_{0}, t, \gamma_{2}\right)\right]
$$

where

$$
\begin{aligned}
I_{J}^{H}\left(f, h_{0}, t, \gamma_{2}\right)= & -\int_{\mathfrak{h}_{p}^{*}} h_{p}^{-\vee(-1) \nu} \int_{a^{*}} I_{J}^{H}\left(\gamma_{2}: J_{2}(t): \mu\right) \sum_{b^{*} \in \hat{J}_{K}} \overline{b^{*}\left(\gamma_{0} \gamma_{2} j_{1}(t)\right)} \\
& \times\left\{\sum_{A \in \operatorname{Car}^{\prime}(G, J)} \int_{G^{A}} \theta\left(J, b^{*}, \mu \otimes \nu\right)(x) d_{G}(x)\right\} d \mu d \nu
\end{aligned}
$$

Proof. The theorem follows from combining Lemmas 4.2 and 4.12 along with (4.14) and then using Lemma 4.1 and $J$ in place of $H$. It also uses the fact that

$$
\sum_{b^{*} \in \hat{J}_{K}} \overline{b^{*}\left(j_{k}\right)} \int_{\mathfrak{h}_{p}^{*}} h_{p}^{-\vee(-1) \nu} \int_{a^{*}} I_{J}^{H}\left(\gamma_{2}: J_{2}(t): \mu\right) \theta\left(J, b^{*}, \mu \otimes \nu\right)(f) d \mu d \nu
$$

converges absolutely.

5. Proof of Lemma 2.5. It suffices to prove the lemma with $s=I$ because of (2.3). If $\lambda_{0} \in F^{+} \cap L_{3}$ is regular, and $t_{i}\left(\lambda_{0}\right)=0$, then there are elements $\lambda^{\prime} \in F^{+} \cap L_{b}$ with $t_{i}\left(\lambda^{\prime}\right)>0$, and so $c\left(I: \lambda_{0}\right)=c\left(I: \lambda^{\prime}\right)=0$. If $\lambda_{0} \in L_{b}^{s}$, let $F^{+}$be a component of $F_{3}^{\prime}$ whose closure contains $\lambda_{0}$. Then $\left\{w F^{+} \mid w \in W^{\prime}\right\}$ are exactly the components of $F^{\prime}$ whose closures contain $\lambda_{0}$, and so $c\left(I: \lambda_{0}\right)$ $=\left(1 /\left[W^{\prime}\right]\right) \Sigma_{w \in W^{\prime}} c\left(I: w F^{+}\right)$.

Suppose first that there is a root $\alpha \in \Phi\left(z_{c}, \dot{l}_{c}\right)$ such that $k y^{-1} \alpha$ is a compact root of $\left(z, \mathfrak{h}_{k}\right)$ and $\left\langle\lambda_{0}, \alpha\right\rangle=0$. Then $s_{\alpha} \in W^{\prime}$, where $s_{\alpha}$ denotes the reflection 
corresponding to $\alpha$, and

$$
\begin{aligned}
& \sum_{w \in W^{\prime}} \operatorname{det} w c\left(w: \lambda_{0}\right)=\frac{1}{\left[W^{\prime}\right]} \sum_{w \in W^{\prime}} \operatorname{det} w \sum_{v \in W^{\prime}} c\left(w: w^{-1} v F^{+}\right) \\
& =\frac{1}{\left[W^{\prime}\right]} \sum_{v \in W^{\prime}} \sum_{w \in W^{\prime} /\left\{I, s_{\alpha}\right\}} \operatorname{det} w\left(c\left(w: w^{-1} v F^{+}\right)\right. \\
& \left.-c\left(w s_{\alpha}: s_{\alpha} w^{-1} v F^{+}\right)\right)=0
\end{aligned}
$$

using (2.4), since $s_{\alpha} \in W\left(Z,{ }^{y k^{-1}} H_{K}\right)$.

Thus we may assume that

$$
k y^{-1} \Phi^{+}\left(\lambda_{0}\right)=k y^{-1}\left\{\alpha \in \Phi^{+} \mid\left\langle\lambda_{0}, \alpha\right\rangle=0\right\}
$$

consists entirely of singular imaginary roots of $\left(\mathfrak{z}, \mathfrak{h}_{k}\right)$. As a consequence, if $\beta_{1}$, $\beta_{2} \in \Phi^{+}\left(\lambda_{0}\right)$, then $\left\langle\beta_{1}, \beta_{2}\right\rangle=0$ and no other element of $\Phi^{+}$is in the linear span of $\beta_{1}$ and $\beta_{2}$. For $\alpha \in \Phi^{+}$, let $H_{\alpha}=\left\{\lambda \in F_{3} \mid\langle\alpha, \lambda\rangle=0\right\}$.Then if $v \in$ $W^{\prime}$ and $\alpha \in \Phi^{+}\left(\lambda_{0}\right), H_{\alpha}$ is the only root hyperplane separating $v F^{+}$and $s_{\alpha} v F^{+}$.

Let $F_{-}=\left\{\lambda \in F_{3} \mid t_{i}(\lambda)<0, i=1, \ldots, l\right\}$. Suppose $w_{1}, \ldots, w_{r}$ are the elements of $W^{\prime}$ satisfying $F_{i}=w_{i} F^{+} \subseteq F_{-}, 1<i<r . \bar{F}_{1} \cup \cdots \cup F_{r}$ is a convex set, bounded by certain hyperplanes $H_{\alpha}, \alpha \in \Phi\left(z_{c}, j_{c}\right)$. Since $\lambda_{0}$ is not in the interior of $\bar{F}_{1} \cup \cdots \cup F_{r}$ which is contained in $F^{-}, \lambda_{0}$ is in at least one hyperplane $H_{\alpha_{0}}$ bounding $\overline{F_{1} \cup \cdots \cup F_{r}}$. Then $s_{0}=s_{\alpha_{0}} \in W^{\prime}$ and $s_{0} F_{i}$ $Z F_{-}, i=1, \ldots, r$. Thus

$$
\begin{array}{rl}
\sum_{w \in W^{\prime}} \operatorname{det} w & c\left(w: \lambda_{0}\right)=\frac{1}{\left[W^{\prime}\right]} \sum_{w \in W^{\prime}} \operatorname{det} w \sum_{v \in W^{\prime}} c\left(w: w^{-1} v F^{+}\right) \\
= & \frac{1}{\left[W^{\prime}\right]} \sum_{w \in\left\{I, s_{0}\right\} \backslash W^{\prime}} \operatorname{det} w \\
& \times \sum_{v \in\left\{I, s_{0}\right\} \backslash W^{\prime}}\left[c\left(w: w^{-1} v F^{+}\right)+c\left(w: w^{-1} s_{0} v F^{+}\right)\right. \\
& \left.\quad-c\left(s_{0} w: w^{-1} s_{0} v F^{+}\right)-c\left(s_{0} w: w^{-1} v F^{+}\right)\right] .
\end{array}
$$

If neither $v F^{+}$nor $s_{0} v F^{+}$is contained in $F_{-}$, the quantity in brackets is zero. Thus we may assume $v F^{+} \subseteq F_{-}$. Then $s_{0} v F^{+} \& F_{-}$and so $c\left(w: w^{-1} s_{0} v F^{+}\right)=c\left(s_{0} w: w^{-1} v F^{+}\right)=0$.

Write $s_{0}=s_{1} \cdots s_{k}$ as a product of simple reflections with $k$ minimal, $s_{i}$ the reflection corresponding to $\alpha_{l(i)}, 1<l(i)<l$. Note that $k$ will be an odd integer. Let $\Gamma_{i} \in \overline{\mathrm{i}_{p}^{+}}$be a semiregular element of $\dot{i}$ corresponding to $\alpha_{l(i)}$, $\mathfrak{h}_{\Gamma_{i}}^{+}=\mathrm{i}$ and $\mathfrak{h}_{\Gamma_{i}}^{-}=\mathfrak{i}_{i}$ the corresponding Cartan subalgebras of $z[8, \S 1.3 .4]$.

Let $z_{i}$ be the centralizer in $z$ of $\left(\dot{j}_{i}\right)_{k}$. Let $\dot{j}_{i}^{+}$be the chamber of $i_{i}^{+}\left(z_{i}\right)$ containing $\Gamma_{i}$. Write $c_{i}\left(s: u F^{+}\right)$for $c_{z_{i}}\left(s: u F^{+}: \mathrm{i}_{i}^{+}\right), s, u \in W\left(z_{c}, \mathrm{i}_{c}\right)$. 
It follows from [1(d)] that

$$
\begin{aligned}
c\left(w: w^{-1} v F^{+}\right)=\sum_{i=1}^{k}(-1)^{i+1}\left(c _ { i } \left(s_{i+1}\right.\right. & \left.\cdots s_{k} w: w^{-1} v F^{+}\right) \\
& \left.+c_{i}\left(s_{i} s_{i+1} \cdots s_{k} w: w^{-1} v F^{+}\right)\right)
\end{aligned}
$$

and

$$
\begin{aligned}
c\left(s_{0} w: w^{-1} s_{0} v F^{+}\right)=\sum_{i=1}^{k}(-1)^{i+1}\left(c _ { i } \left(s_{i} \cdots\right.\right. & \left.s_{k} w: w^{-1} s_{0} v F^{+}\right) \\
& \left.+c_{i}\left(s_{i+1} \cdots s_{k} w: w^{-1} v F^{+}\right)\right) .
\end{aligned}
$$

We claim that for each $i=1, \ldots, k$,

$$
c_{i}\left(s_{i+1} \cdots s_{k} w: w^{-1} v F^{+}\right)=c_{i}\left(s_{i+1} \cdots s_{k} w: w^{-1} s_{0} v F^{+}\right)
$$

and

$$
c_{i}\left(s_{i} \cdots s_{k} w: w^{-1} v F^{+}\right)=c_{i}\left(s_{i} \cdots s_{k} w: w^{-1} s_{0} v F^{+}\right)
$$

so that $c\left(w: w^{-1} v F^{+}\right)=c\left(s_{0} w: w^{-1} s_{0} v F^{+}\right)$. We will show the first equality. The second follows from a similar argument.

To prove the equality, it suffices to show that no hyperplane $H_{\beta}, \beta \in$ $\Phi\left(z_{i \mathrm{C}}, \dot{\mathrm{l}}_{\mathrm{C}}\right)$ separates $s_{k+1} \cdots s_{k} v F^{+}$and $s_{i+1} \cdots s_{k} s_{0} v F^{+}=s_{i} \cdots s_{1} v F^{+}$. Suppose such a root $\beta$ exists. Let $\beta^{\prime}=s_{k} \cdots s_{i+1} \beta$. Then $H_{\beta^{\prime}}$ separates $v F^{+}$ and $s_{0} v F^{+}$. But $H_{\alpha_{0}}$ is the only hyperplane separating $v F^{+}$and $s_{0} v F^{+}$, so that $\alpha_{0}=\beta^{\prime}$, and $\beta=s_{i+1} \cdots s_{k} \alpha_{0}$. Since $\beta \in \Phi\left(\left(z_{i}\right)_{C}, \dot{l}_{C}\right),\left\langle\beta, \alpha_{l(i)}\right\rangle=0$ and $\beta=s_{i+1} \cdots s_{k} \alpha_{0}=s_{i} s_{i+1} \cdots s_{k} \alpha_{0}$. But since $s_{1} \cdots s_{k}$ is a reduced decomposition of $s_{0}$ into simple reflections, this is impossible. Q.E.D.

\section{REFERENCES}

1. Harish-Chandra, (a) A formula for semisimple Lie groups, Amer. J. Math. 79 (1957), 733-760.

(b) Some results on an invariant integral on a semisimple Lie algebra, Ann. of Math. (2) 80 (1964), 551-593.

(c) Invariant eigendistributions on a semisimple Lie group, Trans. Amer. Math. Soc. 119 (1965), 457-508.

(d) Discrete series for semisimple Lie groups. I, Acta Math. 113 (1965), 241-318.

(e) Two theorems on semisimple Lie groups, Ann. of Math. (2) 83 (1966), 74-128.

(f) Discrete series on semisimple Lie groups. II, Acta Math. 116 (1966), 1-111.

2. R. Herb, Fourier inversion on semisimple Lie groups of real rank two, $\mathrm{Ph}$. D. Thesis, Univ. of Washington, Seattle, Wash., 1974.

3. R. Herb and P. Sally, Singular invariant eigendistributions as characters, Bull. Amer. Math. Soc. 83 (1977), 252-254.

4. R. Lipsman, On the characters and equivalence of continuous series representations, J. Math. Soc. Japan 23 (1971), 452-480.

5. D. Ragozin and G. Warner, On a method of computing multiplicities in $L_{2}(\Gamma \backslash G)$ (to appear). 
6. P. Sally and G. Warner, The Fourier transform on semisimple Lie groups of real rank one, Acta Math 131 (1973), 1-26.

7. W. Schmid, On the characters of the discrete series, Invent. Math. 30 (1975), 47-144.

8. G. Warmer, Harmonic analysis on semisimple Lie groups. I, II, Springer-Verlag, Berlin and Now York, 1972.

9. J. Wolf, Unitary representations on partially holomorphic cohomolozy spaces, Mem. Amer. Math. Soc. No. 138 (1974).

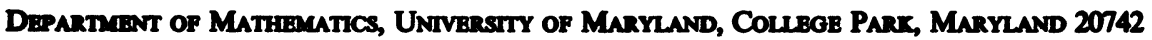

